\title{
ADWICE: Advanced Diagnosis and Warning System for Aircraft Icing Environments
}

\author{
A. TAFFERNER \\ Institut für Physik der Atmosphäre, DLR-Oberpfaffenhofen, Wessling, Germany \\ T. HAUF AND C. LEIFELD \\ Institut für Meteorologie und Klimatologie, Universität Hannover, Hannover, Germany \\ T. HAFNER, ${ }^{*}$ H. LEYKAUF, AND U. VoIGT \\ Deutscher Wetterdienst, Offenbach, Germany
}

(Manuscript received 7 August 2001, in final form 23 July 2002)

\begin{abstract}
This paper describes the design of the Advanced Diagnosis and Warning System for Aircraft Icing Environments (ADWICE) and presents results for two different icing weather situations with typical icing conditions. ADWICE has been in development since 1998 through the joint cooperation of the Institute for Atmospheric Physics at the German Aerospace Center (DLR), the German Weather Service (DWD), and the Institute for Meteorology and Climatology of the University of Hannover (IMUK). ADWICE uses information from different data sources in order to identify atmospheric environments that are potentially hazardous for aircraft icing. Forecast data from the operational Local Model (LM) of the DWD, with a horizontal grid spacing of $7 \mathrm{~km}$ covering the domain of central Europe, are combined with radar data and routine weather observations from the surface station network for this purpose. Algorithms developed at the National Center for Atmospheric Research (NCAR) that take into account different weather scenarios use the LM forecast fields of temperature, humidity, and pressure to provide first-guess icing information at LM grid points. This first-guess field is then subjected to a scenario correction in which a consistency check is performed through the combined use of the radar data and present weather reports. A final correction of the icing volume is achieved through surface observations of cloudiness and ceiling. For all diagnosed icing points the intensity of icing is derived from a formula that provides an adiabatic estimate of cloud liquid water from water vapor saturation mixing ratio at cloud base and forecast mixing ratios from the LM. Results are presented for a typical case of freezing rain and another one in which pilot reports (PIREPs) of icing are available for comparison. These PIREPS have been collected together with other relevant meteorological data during a testing phase from January to May 2001 in which ADWICE has been run in an operational environment at the DWD. Although ADWICE produces plausible icing fields, uncertainty remains with regard to providing an estimate of the icing intensity at a particular flight level. Taking cloud liquid water as forecast by the LM model directly as a measure of icing intensity instead of the estimate provided by the formula, however, produces poor results, as the comparison with PIREPs indicates.
\end{abstract}

\section{Introduction}

In-flight aircraft icing remains a serious threat to the aviation community. To date the number of worldwide known accidents and serious incidents in which icing played a major role exceeds 800 (Mingione 1998). Obviously current protection systems and icing forecasting, which rely mostly on reported icing by pilots and the evaluation of radiosonde ascents, are inadequate to con-

\footnotetext{
* Deceased.
}

Corresponding author address: Dr. Arnold Tafferner, DLR-Institut für Physik der Atmosphäre, Oberpfaffenhofen, 82234 Wessling, Germany.

E-mail: arnold.tafferner@dlr.de trol the threat (Green 1997). Ice accumulation on aerodynamic surfaces while flying in supercooled clouds can result in significant degradation of aircraft performance (e.g., Sand et al. 1984; Politovich 1989; Hauf et al. 1997). Whereas jet aircraft are generally less sensitive to icing due to their greater propulsion power, which makes escape maneuvers more easy to perform, smalland medium-sized turboprop aircraft that operate mostly on short-haul flights and therefore at medium flight levels with higher icing potential have to fully rely on their deicing equipment.

Besides the obvious influence factors for aircraft icing, that is, subfreezing temperature together with a high liquid water content, the droplet size distribution in clouds also plays an important role in the possible ice accumulation rate. Here the so-called supercooled large 
droplets (SLDs), with a size larger than $40 \mu \mathrm{m}$, can be particularly dangerous (Politovich 1989). They have a higher collection efficiency than small droplets due to their larger inertia, causing them not to follow the airstream around the airplane (e.g., Hauf and Brown 1998). Furthermore, when contacting the airframe these SLDs might not freeze immediately but flow along the frame thereby reaching areas unprotected from deicing equipment, at which they can change the shape of the airfoil. In this process ice accumulations can also occur on the underside of the wing (e.g., Hoffmann and Demmel 1990). The resulting new airfoil will have drag, lift, and pitching moment characteristics that may not meet the requirements of the design or the mission (Green 1997). Consequently, a modification of the aerodynamics of the aircraft is possible up to the point of uncontrolled flight. These SLDs are not taken into account in the icing envelopes of the Federal Aviation Regulations (Federal Aviation Administration 1974). To determine an aircraft's potential exposure to icing, meteorological factors need to be considered in conjunction with specific aircraft parameters such as airspeed, flying time in the icing zone, and dimension of the icing profile (e.g., Fuchs and Schickel 1994).

Various weather situations have been identified by scientists to favor the occurrence of aircraft icing in Canada, the United States, and Europe (e.g., Cober et al. 1995; Politovich and Bernstein 1995; McCann 1997; Bernstein et al. 1997; Rauber and Tokay 1991; Hauf and Brown 1998). Generally any uplift of moist air with condensation below $0^{\circ} \mathrm{C}$ can produce supercooled cloud droplets. Therefore warm and cold fronts, convective clouds, and orographic uplift are possible candidates. The amount of supercooled water in such situations cannot be estimated from simple relationships. Several factors are important: temperature, moisture advection, the uplift strength, duration and the area over which the uplift occurs, the presence of cloud ice, aerosol and precipitation particles, ambient moisture, and even turbulence (Nicholls 1987). From this it is obvious that diagnosing and forecasting aircraft icing may be a difficult task. Icing conditions occurring close to the ground are particularly dangerous for aircraft during takeoff and landing. Here freezing rain and freezing drizzle containing SLDs are quite frequent not only in Canada (Stuart and Isaac 1999) and the United States (e.g., Bernstein et al. 1998; Bernstein 2000), but also in Europe (Carrière et al. 2000). Freezing rain and drizzle have however also been reported at altitudes as high as 10000 and $20000 \mathrm{ft}$ MSL, respectively (Jeck 1996).

Of course, the safest way for aircraft would be not to enter the icing regions at all. However, this would require forecasting the amount of supercooled liquid water in clouds occurring in the various weather conditions mentioned above. Cloud liquid water is neither measured directly on a routine basis nor can it be forecast by current weather forecast models with sufficient accuracy (e.g., Guan et al. 2001). It is well known that the position of clouds, including their top and base, are frequently forecast incorrectly by numerical models.

In order to circumvent this difficulty, indirect methods to diagnose and forecast icing conditions have been developed. These methods rely on algorithms that deduce the potential icing threat from measured (mainly radiosonde ascents) or forecast (numerical models) distributions of temperature and humidity (e.g., Thompson et al. 1997a; Carrière et al. 1997). However, as the authors state, although these algorithms have a high probability of detection of icing as verified against pilot reports, they generally produce a high false alarm rate. This situation calls for the use of additional data to reduce the false alarm rate as much as possible. Data fusion concepts have therefore been developed with the aim of localizing potential icing regions from a proper combination of numerical output data, satellite and radar data, as well as surface observations (e.g., McDonough and Bernstein 1999).

Following this concept, the Advanced Diagnosis and Warning System for Aircraft Icing Environments (ADWICE) has been developed since 1998 for application in the European region in joint cooperation with the Institute for Atmospheric Physics at the German Aerospace Center (DLR), the German Weather Service (DWD), and the Institute for Meteorology and Climatology of the University of Hannover (IMUK). During its development phase ADWICE has been verified with data collected during the European Research for Aircraft Ice Certification (EURICE) field phase (Hauf et al. 1997) by Leifeld (1999). These first results were promising. By that time, however, it was clear that a longer testing phase with more data was necessary as well were improvements to the algorithms. After having introduced a data fusion concept for model forecast, and radar and surface observation data, ADWICE was implemented preoperationally at DWD in January 2001 and was run from January through May 2001. This testing phase aimed at checking the usefulness of ADWICE in an operational environment, to gather experience of the performance of ADWICE within different synoptic situations, and to collect output from ADWICE together with all available weather data for future detailed interpretation. Also pilot reports of icing were collected during these months for posterior verification of the icing diagnostics. This paper describes the design of the ADWICE system and presents first results from two different synoptic cases.

\section{Overview of the ADWICE system}

ADWICE combines various data sources in order to identify icing environments. Figure 1 illustrates the processing of the data. The system starts with forecasts of the DWD's Local Model (LM; Doms and Schättler 1999) to calculate a "first guess icing" by applying a slightly modified version of the National Center for Atmospheric Research/Research Applications Program 


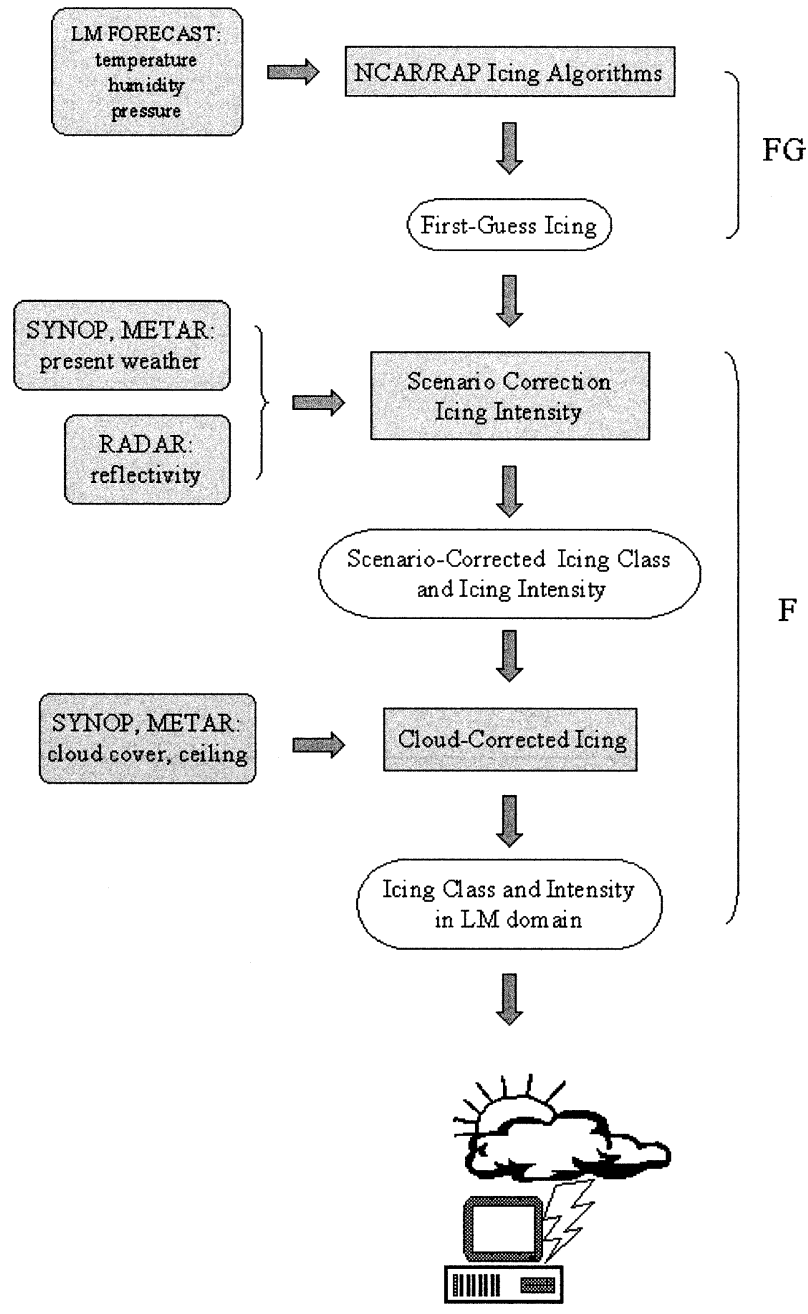

FIG. 1. Data processing in ADWICE. (left) Input data and (right) icing algorithms and icing data. FG = first-guess part of ADWICE; $\mathrm{F}=$ fusion part of ADWICE. The workstation symbol is the logogram of MAP from DWD.

(NCAR/RAP) algorithm [Thompson et al. (1997a); discussed in detail in section 3]. This first-guess icing information in the LM grid distinguishes four different meteorological regimes: general, unstable, warm stratus, and freezing rain (explained in section 3 ). The firstguess field is then combined with observational data. Surface weather reports (SYNOPs) and routine weather reports for aviation (METARs) are used in combination with radar reflectivity data from the European radar network assembled at DWD to check the first-guess icing field. This so-called scenario correction confirms or rejects the first guess depending on simultaneous observations from radar, SYNOPs, and METARs. Also new icing points are introduced if there is a clear indication for icing conditions at the surface or up to a certain height from the surface reports. Furthermore, SYNOP and METAR reports of cloud cover and ceiling are used to examine the diagnosed icing field for cloudless re- gions, providing a "cloud-corrected icing." Last, icing intensity is calculated from water vapor saturation mixing ratio at cloud base and mixing ratio within the cloud as forecast by the LM. All of these procedures are outlined in detail in section 3 .

The two icing fields, icing class and icing intensity, are output from ADWICE on LM model levels as well as on selected pressure levels representative of flight levels. They can be displayed graphically as shown later and can also be transferred for visualization and overlay with other fields to the Meteorological Application and Presentation system [MAP; Kusch and Pagoda (1995), indicated at the bottom of Fig. 1]. MAP is the routine data processing and display system for regional weather dissemination at the DWD. Routine observational data, satellite, radar, and lightning observations as well as model forecasts are continuously transmitted from the DWD to MAP workstations at the regional forecasting offices. MAP has been specifically designed to allow a quick display of data, to overlay graphics, and to calculate and show derived products like nowcasting of thunderstorms, sounding evaluation, METARs along flight corridor, and many others. The forecaster is therefore able to have an overview of the weather state and issue nowcasts and warnings to users (e.g., the aviation community).

The icing information available in MAP can be used by forecasters in two ways. The first guess icing field that is available soon after the end of an LM forecast run, that is, about $4 \mathrm{~h}$ after the initialization times 0000 and 1200 UTC, can be used by the forecaster in advance of the actual icing event as background information on what could happen in the future. Later on, the final icing diagnosis can be used to issue warnings to the air traffic community. The final icing fields are updated every hour thus allowing an almost continuous synopsis of the icing threat (for operational aspects see section 6).

\section{Use of data sources}

\section{a. Numerical model output data}

Forecast profiles of temperature and humidity from the LM serve as input to the NCAR/RAP icing algorithm (Thompson et al. 1997a), which distinguishes four icing regimes to be discussed in the following.

\section{1) Freezing Rain Regime}

Figure 2a sketches typical profiles of temperature and dewpoint temperature in a freezing rain scenario. On top of a warm layer with temperatures above $0^{\circ} \mathrm{C}$ - the so-called warm nose-a relatively moist layer exists where precipitation forms via the ice-phase process. Precipitating particles falling through the warm nose melt and become supercooled as they fall into the subfreezing layer below. Because this layer is also relatively humid the particles do not evaporate. A lower threshold of $80 \%$ 


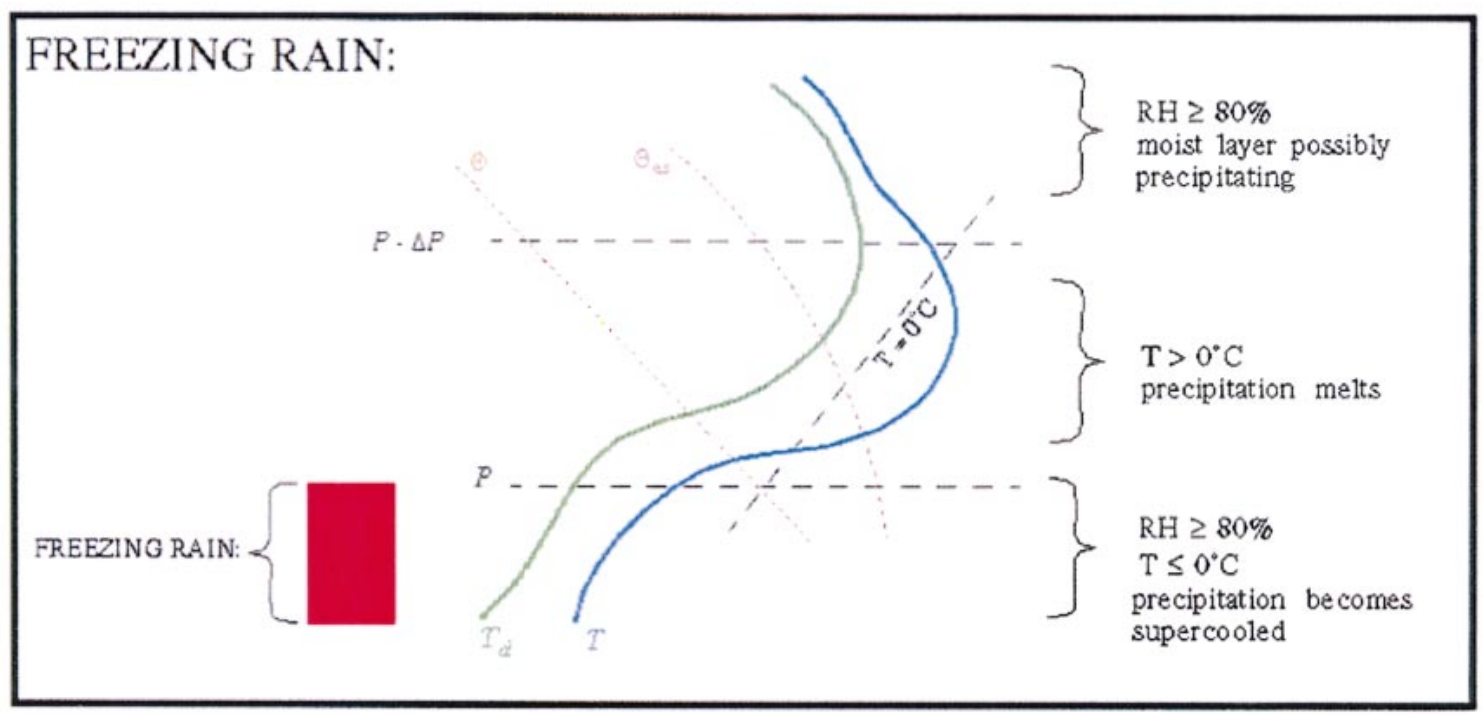

a

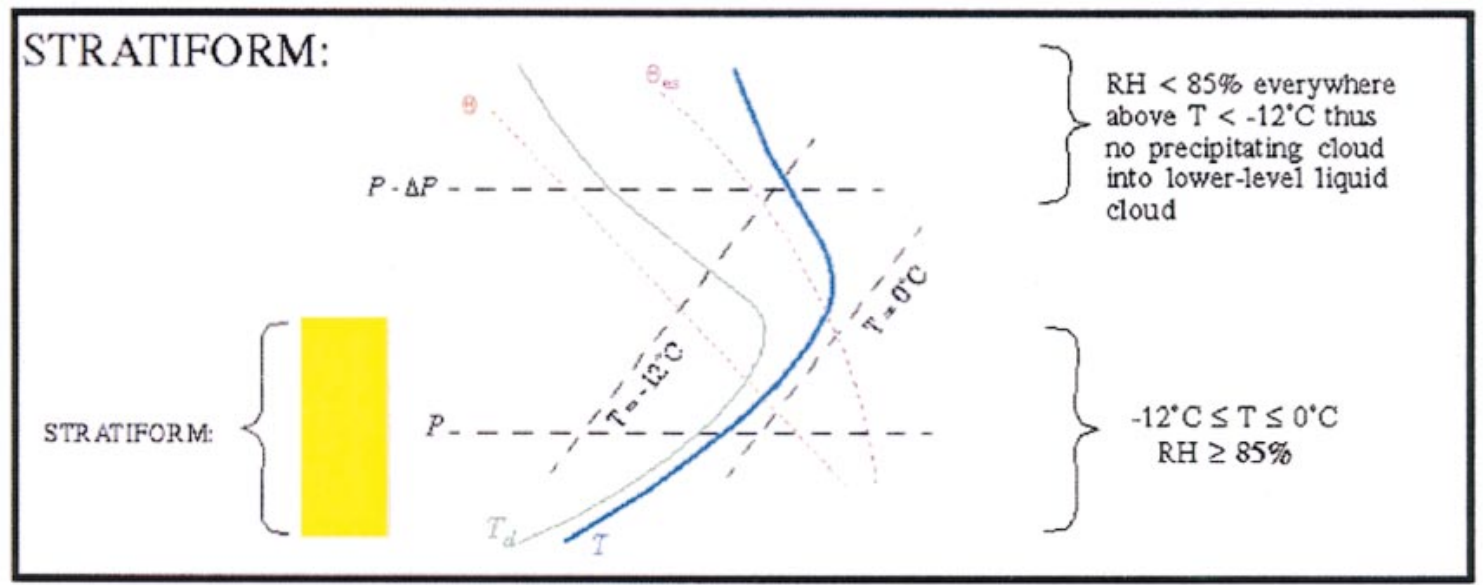

b

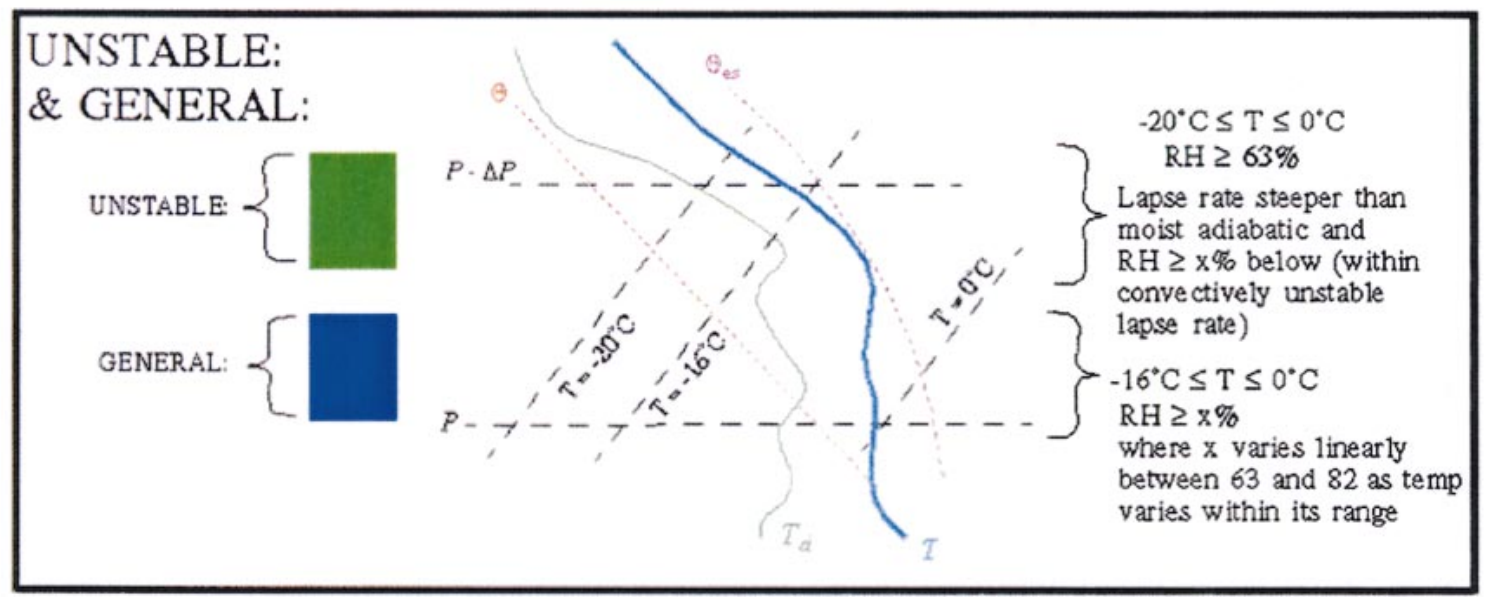

C

FIG. 2. NCAR/RAP icing algorithm, from Thompson et al. (1997b).

has been prescribed for relative humidity to the moist layers on top and underneath the warm nose. This value has been set according to radiosonde observations in the vicinity of icing pilot reports (PIREPs) (Forbes et al. 1993). The supercooled particles below the warm nose will instantly freeze when contacting a surface temperature that is below freezing, be it the ground or an airplane. Furthermore, supercooled precipitating parti- 
cles consist of large raindrops with a high collection efficiency. If this icing scenario occurs close to the ground it can also cause a very dangerous situation. Here airplanes are typically in the approach or landing phase where a performance penalty due to icing might lead to a crash or force exiting maneuvers that are not easy to perform. Of course, aircraft prepared for takeoff (ground icing) can also be affected in such a situation.

\section{2) WARM STRATUS REGIME}

Figure $2 \mathrm{~b}$ sketches typical profiles of temperature and dewpoint temperature in the case of a stratiform cloud. There is a moist layer with a prescribed relative humidity greater than $85 \%$ within a temperature range from $-12^{\circ}$ to $0^{\circ} \mathrm{C}$ topped by an inversion, above which the air is relatively dry (relative humidity less than $85 \%$ ). Thereby it is assumed that no precipitation from above is falling into the subfreezing layer, which could deplete supercooled droplets. We changed the original algorithm slightly by allowing clouds, that is, a relative humidity greater than $85 \%$, between cloud top and the $-50^{\circ} \mathrm{C}$ level. This takes into account that a nonprecipitating cirrus cloud will not influence the stratus cloud deck at low levels. In contrast to the freezing rain scenario where precipitation forms through the ice-phase process, here freezing drizzle can form within the moist layer via condensation-collision-coalescence, the socalled "warm rain" process (Huffman and Norman 1988; Strapp et al. 1996). This icing scenario can be also quite dangerous for aircraft. The lower-tropospheric cloud layer can be quite deep and spread out over large horizontal distances. When the cloud base is close to the ground and/or freezing drizzle reaches the ground, escape maneuvers are successful only in an upward direction, which may be impossible for turboprops when the rate of climb is already reduced by ice accumulation (Sand et al. 1984). In fact, studies on freezing precipitation show that this warm-rain process seems to occur more often than when freezing precipitation forms via the ice-phase process (Strapp et al. 1996; Rauber et al. 2000).

\section{3) Unstable REGIME}

An unstable regime is depicted in Fig. 2c. Here icing is diagnosed when the following conditions are met at the same time: first, at a certain level the temperature is between $-20^{\circ}$ and $0^{\circ} \mathrm{C}$ and the relative humidity is greater than $63 \%$, and second, below this level an unstable (at least conditionally unstable) layer exists in which the maximum humidity is at least $x \%$, with $x$ varying linearly between $63 \%$ and $82 \%$ as temperature varies between $-20^{\circ}$ and $0^{\circ} \mathrm{C}$.

This scenario mimics an icing situation within relatively warm convective clouds where vertical moisture transport can provide abundant supercooled droplets because the production rate can exceed the depletion rate in updrafts (Rauber and Tokay 1991; Politovich and Bernstein 1995). In particular this can be a dangerous situation when the clouds are embedded in a stratus cloud. The inversion on top of the stratus deck hinders potentially deep ascent of the cloud to colder temperatures thereby suppressing ice crystal production and subsequent depletion of supercooled liquid water. Hauf and Schröder (1998) have found relatively high icing rates up to $3.5 \mathrm{~mm} \mathrm{~min} \mathrm{~min}^{-1}$ in embedded convective clouds during research flights in Germany. Pilots flying in a stratus deck cannot see embedded convective clouds, making this scenario of icing difficult to detect. This is of course only true if the icing threat in the stratus cloud is low, which might not always be the case. In order to take embedded convection into account we have changed the original version of the NCAR/RAP algorithm and now allow unstable icing regimes within warm stratus regime; that is, in the program code we allow diagnosed "warm stratus" to be overwritten by "unstable" if the latter is diagnosed after the first within a vertical grid column.

\section{4) General Regime}

If none of the above icing regimes can be diagnosed, there is still a chance for icing whenever temperature and humidity lie within a certain range. Here thresholds of $-16^{\circ}$ and $0^{\circ} \mathrm{C}$ are assigned for temperature with humidity varying as in the unstable regime (indicated in Fig. 2c). No clear identifiable process leading to icing can be distinguished in this regime.

\section{5) FURTHER REMARKS}

The NCAR/RAP algorithm uses temperature and humidity thresholds derived from a multitude of radiosonde observations in the vicinity of pilot reports of icing according to Forbes et al. (1993). They found a mean value of relative humidity of $82 \%$ with a standard deviation of $19 \%$ (therefore the thresholds $63 \%$ and $82 \%$ in the algorithm). Thompson et al. (1997a) used forecast model profiles of temperature and humidity from numerical models that might not necessarily correspond with observed radiosonde soundings due to errors in physical parameterizations, initialization, and others. Another complication resides in the model's grid resolution, which is usually too coarse to resolve clouds. Consequently model relative humidities are not in accord with real atmosphere relative humidities in cloudy regions except when proper parametrizations provide the necessary ajustment. With increasing resolution one might get closer to the $100 \%$ limit as moisture maxima and gradients are inherently smoothed in coarser grids. However, the greater problem is the lower threshold. Taking it too high assumes that we rely on the numerical model to forecast the position and extent of clouds correctly. Taking it too low has the effect of expanding the areas of icing, thereby increasing the false alarm rate. 
The aim therefore is to find an ideal compromise between capturing as much of the icing observations as possible - high probability of detection (POD)—on one hand, while keeping the false alarm rate as small as possible on the other hand. Thompson et al. (1997a) did not succeed in fine-tuning temperature and humidity thresholds in order to maintain a high POD while decreasing substantially the impacted area. They concluded that the icing diagnostics provided by the algorithm are best used as a first guess field and additional data like surface and remote sensing data should be taken into account. Also they suggested that using cloud liquid water from model output directly should improve the icing diagnostic. However, cloud liquid water forecasts from today's operational models are of poor quality (e.g., Guan et al. 2001). Because of their coarse resolution, imperfect moisture initialization, and imperfect cloud physics, one cannot expect realistic cloud water forecasts for the near future (discussed further in section 4b).

Summarizing, we can state that the NCAR/RAP algorithm provides a first-guess icing field that has to be refined with observational data. As explained by Thompson et al (1997a), its strength is that it gives the physical reason for icing, which is very important to the forecaster and is therefore superior to simple yesno predictions.

\section{b. Radar data}

Conventional radars cannot detect most of the icing regions since the wavelength of the radars within the European radar network (assembled routinely by DWD) is about $5 \mathrm{~cm}$ long whereas most icing occurs in cloudsized droplets. Even SLDs up to the size of freezing drizzle $(400 \mu \mathrm{m})$ can generally, depending in the distance from the scattering object, not be seen. Nevertheless radar data can be used as important information to confirm or reject the first guess icing field particularly in combination with surface observations. A threshold of $19 \mathrm{dBZ}$, which is the upper bound of the lowest intensity range in the European radar composite, is set for certain checks in the scenario correction (see section $3 d)$. For example, precipitating snow or drizzle can be detected between 0 and $19 \mathrm{dBZ}$. Given a horizontal resolution of $2 \mathrm{~km} \times 2 \mathrm{~km}$ for the radar data, an average of the four nearest pixels surrounding an LM point is taken as representative for that grid point.

\section{c. SYNOP and METAR data}

Hourly surface observations of present weather, cloud cover, and ceiling from SYNOPs and METARs are used to examine the first guess icing field. As about $95 \%$ of the automated weather stations throughout central Europe do not report significant weather, we only use manned weather stations for the analysis. For carrying out the checks against SYNOP and METAR reports,

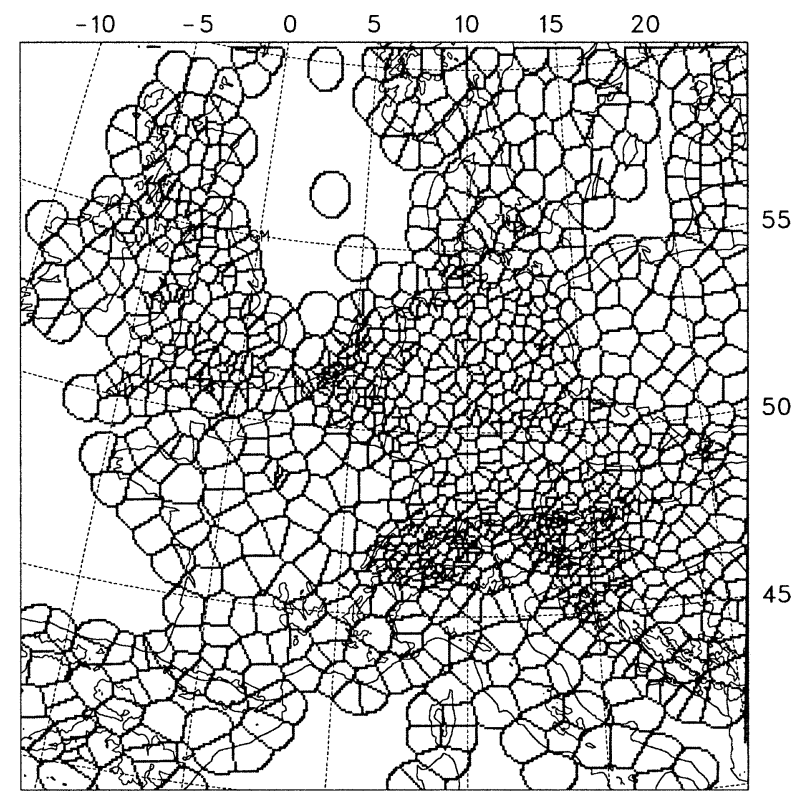

FIG. 3. Influence regions of surface stations reporting at 1200 UTC 23 Dec 1999 when a maximum influence radius of $70 \mathrm{~km}$ is assumed.

one is faced with the problem of determining how representative the reports are for the immediate area surrounding the observation location. A station reporting on the Atlantic can be treated as representative for a wider area than one lying in a mountain valley. To do it correctly, one would have to investigate every station for different weather scenarios in order to find out its representative influence radius. This, however, is an elaborate task. Here we profit from the high station density in central Europe, which makes it possible to find a "nearby" station for almost every grid point. Figure 3 shows the influence regions of all manned stations reporting at a particular time throughout the LM domain for a maximum prescribed influence radius of $70 \mathrm{~km}$. This value seems rather large; however, one can see that most of the stations over land are influenced by a nearby station within a distance of much less than $70 \mathrm{~km}$. For comparison, note that the circle surrounding a station in the North Sea indicates a $70-\mathrm{km}$ influence region since there are no other nearby stations. Only over the seas there are not enough stations to cover all the grid domain.

\section{d. Scenario correction}

Icing forecasts based solely on humidity and temperature thresholds generally produce a high false alarm rate, which can, however, be reduced if consistency checks are carried out against surface observations and radar data. Such a "scenario correction" of the first guess field is performed as follows. Whenever there is a radar and/or surface observation "influencing" an LM grid point, the corresponding icing forecast by the first- 
TABLE 1. Scenario correction for icing regimes. Here, fgss stands for first-guess icing in one of the categories (refer to Fig. 2): ge, general; co, unstable; ws, warm stratus; or fz, freezing rain. Reflev means radar reflectivity level, ww is present weather reported from a manned surface station in WMO specified coding, and ice flag is a number used to extract final icing volume [its meaning is commented upon in the last column to the right in the table (sfc-obs stands for surface observation of SYNOP or METAR)]. The following surface observations are distinguished: 56 (drizzle, freezing, slight), 57 (drizzle, freezing, moderate or heavy), 66 (rain, freezing, slight), 67 (rain, freezing, moderate or heavy), 79 (ice pellets), and $50 \leq \mathrm{ww} \leq 99$ means that the surface observation is within one of the following groups: rain, solid precipitation, showery precipitation, or precipitation with current or recent thunderstorm, $<60$ means either no precipitation or precipitation in the form of drizzle only, $<50$ excludes all kind of precipitation, a dash stands for no observation or observation not taken into account, and no obs means that a station is reporting but not issuing a weather observation.

\begin{tabular}{|c|c|c|c|c|}
\hline Fgss & Reflev (dBZ) & Ww & Ice flag & First guess confirmed or rejected \\
\hline $\mathrm{fz}$ & $>0$ & $56,57,66,67$ & 34 & Confirmed by radar and sfc-obs \\
\hline $\mathrm{fz}$ & - & $56,57,66,67$ & 24 & Confirmed by sfc-obs \\
\hline $\mathrm{fz}$ & - & $50 \leq \mathrm{ww} \leq 99$ and $\mathrm{ww} \neq 56,57,66,67$ & 64 & Rejected fgss at surface by sfc-obs \\
\hline $\mathrm{fz}$ & $>0$ & - & 14 & Confirmed by radar \\
\hline $\mathrm{fz}$ & 0 & $<50$ & 74 & Rejected by radar and $\mathrm{sfc}-\mathrm{obs}$ \\
\hline $\mathrm{fz}$ & - & $<50$ & 64 & Rejected by sfc-obs \\
\hline ws & $0 \leq$ reflev $\leq 19$ & $<60$ or no obs & 33 & Confirmed by radar and sfc-obs \\
\hline ws & - & $<60$ or no obs or 66,67 & 23 & Confirmed by sfc-obs \\
\hline ws & $0 \leq$ reflev $\leq 19$ & - & 13 & Confirmed by radar \\
\hline ws & $>19$ & $60 \leq w w \leq 99$ & 73 & Rejected by radar and sfc-obs \\
\hline ws & $>19$ & - & 53 & Rejected by radar \\
\hline ws & - & $60 \leq \mathrm{ww} \leq 99$ & 63 & Rejected by sfc-obs \\
\hline co & - & $56,57,66,67,79$ & 22 & Confirmed by sfc-obs \\
\hline ge & - & $56,57,66,67,79$ & 21 & Confirmed by sfc-obs \\
\hline ge & - & $<50$ or no obs & 21 & Confirmed by sfc-obs \\
\hline
\end{tabular}

guess algorithm gets attached to one of the following flags: 1) confirmed by radar and surface observation, 2) rejected by radar and surface observation, 3 ) confirmed by radar, 4) rejected by radar, 5) confirmed by surface observation, and 6) rejected by surface observation. "Influencing" with respect to surface observations means that this station is the closest reporting station at that time within an allowed maximum distance of 70 $\mathrm{km}$ (see the example in Fig. 3). Combinations of forecast icing regime (fgss), radar reflectivity level (reflev), weather reports from manned stations (ww), and the resulting flags are summarized in Table 1. The assumptions for setting the flags are listed below. All checks within a particular regime are carried out sequentially until a particular condition is met. If that is the case, the following checks within this particular regime are skipped. After all checks have been performed the final icing volume is then extracted from a proper combination of flags as described at the end of this section.

\section{1) FREEZING RAIN (FZ)}

Here the following checks are consecutively performed (cf. Table 1). If there is a radar reflectivity greater than $0 \mathrm{dBZ}$ together with a station report of freezing rain or drizzle [World Meteorological Organization (WMO) code 56, 57, 66, or 67] for a gridpoint location, all freezing rain points in the vertical up to the lower level of the warm nose are verified. Each of these points gets attached the flag " 34 ," which stands for freezing rain confirmed by radar and surface observation. In this case the proposed scenario is very likely to exist, as the first guess is verified by two independent observations. Note that we also allow for freezing drizzle and not only for freezing rain. The observation might be inaccurate and it would be dangerous to be too restrictive. Also in the case of only a surface report without a simultaneous radar observation the first guess is confirmed up to the level of the warm nose, then attaching the flag "24." Although the radar should see freezing rain, we have to take into account that the grid point might be outside the radar composite or cannot be seen due to shadowing effects in mountainous regions. If there is a precipitation report at the surface other than freezing rain or drizzle, that is, ww between 50 and 99 but not 56, 57, 66, or 67 , the first guess of freezing rain is rejected for the surface only, that is, for the lowest LM level, but is kept for the LM levels above (flag 64). The rain or drizzle droplets might be supercooled aloft as indicated by the forecast thermodynamic structure (otherwise the algorithm would not have diagnosed "fz" for some points in the vertical grid column). Note that ice pellets, which are a strong indication for the existence or freezing rain aloft (Bernstein 1996; Bernstein et al. 1998), are included in this check. However, ice pellets are, as yet, not treated separately from other precipitation forms at the ground. This could be done in a more sophisticated version of the scenario correction (discussed further at the end of this section). In the next check we admit a radar reflectivity to confirm the icing forecast even without an available surface report (flag 14). The respective grid point might be located outside the allowed influence region of a station or the respective station is not reporting at that time. On the other hand the first guess is rejected if a reporting station at that time does not issue any kind of precipitation ( ww $<50$ ), be it with a concurrent radar report of no reflectivity (flag 74) or irrespective of the radar report (flag 64). Note however, 
that in the case of an existing reflectivity $>0$, flag 14 will have been identified before.

\section{2) WARM STRATUS (WS)}

A first check is performed to see whether there are simultaneous measurements of radar reflectivity in the range from 0 to $19 \mathrm{dBZ}$ and surface observations of no precipitation or drizzle only (ww $<60$ ) that confirm this regime (flag 33). Here a threshold of $19 \mathrm{dBZ}$ is set because drizzle droplets can occur within a warm stratus cloud that can be seen by radar at close distances. Also independent surface or radar observations confirm this regime (flags 23 and 13). For the surface observation we also allow here for freezing rain $(\mathrm{ww}=66,67)$ because of possible inaccurate observations. Next we check whether there is a radar measurement with a reflectivity level greater than $19 \mathrm{dBZ}$ together with a surface observation of precipitation other than drizzle, that is, $w w \geq 60$ (flag 73). This would indicate that there is a cloud with precipitation-sized particles other than drizzle, indicating that the first guess is wrong. Finally we check for a radar reflectivity greater than $19 \mathrm{dBZ}$ and for a precipitation type other than drizzle independently as there might not exist a station report or a radar measurement at that grid point at that time. Such observations also reject the first guess (flags 53 and 63), but of course only if none of the conditions above has been verified.

\section{3) Unstable Regime (CO)}

This first guess cannot be rejected by any type of radar or surface observation. Within the unstable regime we can have precipitating or nonprecipitating clouds and therefore might have a radar reflectivity or not, as well as any kind of surface report about precipitation, including none. Therefore, we only look for reports of freezing rain or drizzle that confirm icing conditions aloft (flag 22).

\section{4) General Regime (GE)}

Here no physical process can be identified for icing conditions to occur. Therefore, from surface or radar observations we cannot falsify this regime. As above, we only take reports of freezing rain or drizzle to confirm this regime (flag 21).

\section{5) Surface obSERVATIONS}

The procedure described above in sections $3 \mathrm{~d}(1)-$ $3 \mathrm{~d}(4)$ is followed afterward by yet another check against surface observations. Here the first guess field is changed and/or new icing points are introduced when there are reports of freezing rain $(\mathrm{ww}=66,67)$, ice pellets $(\mathrm{ww}=79)$, or freezing drizzle $(\mathrm{ww}=56,57)$ at the ground that have not been taken into account during the checks above. This step is necessary as the first guess might miss these dangerous icing types at some grid points due to an inaccurate or even an totally wrong forecast and a pure confirmation or rejection of the first guess would not detect these observation points. Therefore, such surface reports imply the flags 44 (observed freezing rain or ice pellets) and 43 (observed freezing drizzle) for LM grid points at the surface within the influence region of these stations. Furthermore, grid points above these surface stations are checked for possible icing points from the first guess. For example, sometimes we find a first guess of freezing rain for grid points above the surface but not for the lowermost layers. This can happen, for example, if the temperature at the lowest model levels is forecast to be above freezing. In this case the missing freezing rain points are filled in (flagged by 44). This correction is also in agreement with the so-called stovepipe algorithm (Bernstein 1996), which is based on the assumption that the observation of freezing rain or ice pellets at the ground is indicative of the occurrence of SLDs within a certain depth above the ground. In the case of reported freezing drizzle together with an icing zone identified by the first guess for layers above ground, we fill the remaining points in the vertical from the ground up to the icing zone with freezing drizzle points (flagged by 43). Here there is obviously freezing precipitation out from the cloud and therefore the zone from cloud base downward has to be identified as an icing zone too. Note, however, that if freezing precipitation is observed but not forecast for any layer above the ground, freezing rain flags cannot be extended to layers above ground as in that case we do not have reliable information about the vertical depth of the icing zone. In that case only the lowest LM grid point gets assigned a flag indicating icing due to observed freezing rain or drizzle (44 or 43 ).

\section{6) EXTRACTION OF ICING POINTS}

After having set all the flags, the final icing analysis can then be extracted by a proper combination of flags; that is, one can decide whether to take only certain points in the grid volume that are definitely icing or noicing points, or allow more uncertainty. Definite icing points could be considered as those where the first guess is confirmed both by radar and surface observations (flags 34, 33) with the inclusion of surface observations not forecast (flags 43, 44). Presently all first guess values not rejected either by radar or surface observation are taken for the final icing grid (flags 34, 24, 14, 33, 23, 13,22 , and 21) together with the icing grid points introduced by observations (flags 43,44 ). Rejected icing points are considered as no icing. Future verifications of icing forecasts with the aid of pilot reports will suggest the best compromise. Also the future use of satellite data (section 5) from the Meteosat Second Generation (MSG) satellite opens further possibilities for identification of the icing volume. 
The above scenario correction also allows the introduction of possibility estimates for the occurrence of icing following the approach of the NCAR/RAP Integrated Icing Diagnostic Algorithm (IIDA; McDonough and Bernstein 1999). For example, a first guess of freezing rain confirmed by radar and surface observations gives a high probability of this type from the ground up to the level of the warm nose. However, in this version of ADWICE, we want to learn first from this basic approach before more sophisticated measures are undertaken. Whatever they will be, the design of the scenario correction is such that it allows for the inclusion of additional data and additional-maybe more complex-conditional dependencies among the data.

\section{e. Cloud cover and cloud base}

Following the scenario correction, surface observations of cloud cover and cloud base from SYNOP and METAR data are used to further reduce the scenariocorrected first guess icing volume. Whenever there is no cloud observation there will be no icing in the vertical column above the observing station. Currently we prescribe a threshold of $4 / 8$ for "cloudless" observations. This threshold has been determined subjectively from comparing cloud and icing observations with forecasted icing scenarios. In a similar way, an icing point is rejected if there is an icing prediction below observed cloud base, provided that it is not of the freezing rain or freezing drizzle variety.

\section{f. Determination of the icing intensity}

The icing product described so far resembles a yesno icing forecast together with the meteorological reason for icing, which we called icing regime. An estimation of icing severity is necessary too. To take this into account we would have to know the liquid water content within the cloud. As we do not have these observations, the liquid water content must be either estimated indirectly by temperature and humidity or taken directly from model output. Studies in this direction with the fifth-generation Pennsylvania State UniversityNCAR Mesoscale Model (MM5) have been promising for an icing case in the United States (Reisner et al. 1998). However, other studies indicate that model forecasts in this respect are quite weak, as already mentioned above (Guan et al. 2001). To date we have refrained from taking cloud water forecasts from the LM model because the cloud physics package currently implemented in the LM is not sophisticated enough to give a reliable measure of cloud liquid water; for example, cloud ice is not yet implemented operationally. First tests with the forecast cloud liquid water from the LM give evidence that this quantity does not seem to provide a good measure about the icing intensity in icing zones (section 4b). It is also known that a proper representation of small-scale cloud physics needs a grid spacing of 2 $\mathrm{km}$ or less (Reisner et al. 1998) whereas the LM's grid resolution is currently $7 \mathrm{~km}$. As the LM is continuously being improved both in model physics, data analysis, and resolution, one might expect that sooner or later supercooled liquid water will be taken directly from the model output as a measure of icing severity for those areas where the above procedures have diagnosed an icing threat. Relying solely on forecast liquid water without going through the data processing as described in this paper, however, would certainly be misleading in many cases. It is well known that precipitation often is forecast incorrectly, not only due to model deficiencies, but also due to the lack of data in the mesoscale, especially as concerns the exact position and state of clouds.

Currently we prescribe the icing intensity "severe" to all diagnosed points of freezing rain and freezing drizzle (flags 34, 24, 14, 43, 44; see Table 1). For the remaining points within the regimes general, unstable, and warm stratus we deduce the icing intensity from estimations of liquid water content (LWC) in clouds by using the following formula (for the derivation see the appendix):

$$
\mathrm{LWC}_{i+1}=1 / R\left[\left(M_{i}-M_{i+1}\right) / T_{i}\right] p_{i} .
$$

Here $\mathrm{LWC}_{i}$ is the liquid water content $\left(\mathrm{g} \mathrm{m}^{-3}\right)$ at $\mathrm{LM}$ level $i, M_{i}$ is the mixing ratio at level $i\left(\mathrm{~g} \mathrm{~kg}^{-1}\right), T_{i}$ is the temperature at level $i(\mathrm{~K}), p_{i}$ is the pressure at level $i$ in pascals, and $R$ is the gas constant for dry air (287 $\mathrm{J} \mathrm{kg}^{-1} \mathrm{~K}^{-1}$ ). Here, $M, p$, and $T$ are forecast variables of the LM. Index $i$ is increasing upward.

The above equation is integrated upward from the cloud base, which is defined as the lowest level of vertically coherent layers of diagnosed icing in one of the four regimes. There can be more than one such "cloud" in the vertical. At cloud base the saturation mixing ratio is taken for mixing ratio $M_{i}$. This formula assumes that the available water vapor at cloud base is partly condensed to cloud water within the cloud layers. Precipitation, freezing, and mixing are not taken into account. Thus the formula provides an adiabatic estimate of the available cloud water provided by the upward motion transport within the cloud. This assumption can be quite wrong within slantwise lifting like in moist conveyor belts where the moist air mass is gradually lifted as a whole. For example severe icing conditions are frequently found ahead of warm fronts (Bernstein et al. 1997, 1998). Considering that we do not have other information of physical properties within a particular cloud, the above formula provides at least a rough estimate of icing intensity. From the liquid water content an icing intensity based on normalized icing degrees can be deduced. Thereby the icing degree is related to the ice accumulation on a cylinder with a 3-in. diameter within an airstream of velocity $320 \mathrm{~km} \mathrm{~h}^{-1}$ (Air Weather Service 1980). It is distinguished between cumuliform and stratiform clouds. For cumuliform clouds a mean volume diameter of $17 \mu \mathrm{m}$ is taken as a basis whereas 
TABLE 2. Icing categories for given LWC $\left(\mathrm{g} \mathrm{m}^{-3}\right)$ for stratiform (strat) and cumuliform (cumul) clouds (following Lewis 1947).

\begin{tabular}{cccl}
\hline \hline LWC strat & LWC cumul & Icing category & Symbol \\
\hline$<0.11$ & $<0.07$ & Trace & \\
$0.11-0.69$ & $0.07-0.50$ & Light & LGT \\
$0.69-1.33$ & $0.50-1.0$ & Moderate & MOD \\
$>1.33$ & $>1.0$ & Severe & SEV \\
\hline
\end{tabular}

for stratiform clouds $14 \mu \mathrm{m}$ is assumed. The icing intensities are then related to the liquid water content as listed in Table 2 (Lewis 1947).

One problem with distributing available water vapor as condensed liquid water over several LM vertical layers is readily apparent. The more layers we have within a cloud, the smaller will be the condensate in one layer. When comparing against PIREPs, this makes it difficult for finding a representative value for a respective flight level. We will come back to this problem in a description of an icing case in section $4 \mathrm{~b}$. For the two cases discussed is sections $4 \mathrm{a}$ and $4 \mathrm{~b}$, "severe" icing intensity is diagnosed in $1 \%$ and $5 \%$, "moderate" in $10 \%$ and $6 \%$, and "light" in about $89 \%$ of all icing points. Other studies have shown that nearly $65 \%$ of all icing is reported as light and about $2 \%$ as severe (Schultz and Politovich 1992; Kane et al. 1998). Therefore, our approach gives reasonable values.

\section{First results}

\section{a. The freezing-rain case from 23 December 1999}

On 23 December 1999 a warm front progressed over Germany from northwest to southeast. The MAP plot (Fig. 4) shows significant weather observations from 1200 UTC. A long band of freezing precipitation extends from the Baltic Sea toward the Rhine-Main area into France. To the south of this band over central and southern Germany there is full cloud cover but no precipitation. Behind the freezing-rain band on the warm northern side of the front precipitating rain is reported in a small band. The radar observation for this time (Fig. 5) shows a reflectivity greater than $37 \mathrm{dBZ}$ within the precipitating regions and weaker reflectivity along the edges of the rainband. Note that the domain of the radar composite does not fully cover the LM domain. Thin lines in the right-hand corners at the bottom and top mark outer boundaries of the composite to the northeast and southeast. The 12-h forecast of the LM starting at 0000 UTC on 23 December 1999 leads to the first guess icing field for the surface as shown in Fig. 6a. Every LM model grid point for which icing is forecast is marked by a colored dot: red, freezing rain; yellow, warm stratus; green, unstable; and blue, general icing regime. Because of the high horizontal grid resolution of $7 \mathrm{~km}$ some areas appear as uniformly colored patches. Let us start the discussion with the freezing rain scenario.

The first guess is able to catch the freezing rainband seen in the observations at the right position; however, there are also regions with freezing rain to the east of this band that are not occurring in the observations, that is, in central Germany, in the Czech Republic, and along the east coast of the Baltic Sea. Figure 6b shows the icing diagnostic after radar and surface observations from SYNOP and METAR data have been fused with the first guess field by applying the scenario correction and after cloudless grid points have been removed. Here, digits 1, 2, and 3 in icing areas denote icing intensities of light, moderate, and severe, respectively. Obviously the overforecast regions of freezing rain have been eliminated as well as most of the icing areas of the general (blue) and warm stratus (yellow) type over eastern Europe. Before we discuss these other icing types we inspect the vertical extension of the freezing rain. ADWICE forecasts freezing rain not only for the ground, but up to the level of the warm nose as seen in the temperature soundings. Figure 7 displays observed (blue) and LM forecast (red) soundings of temperature and dewpoint temperature for the German radiosonde station Greifswald. This station is well within the freezing-rain band (cf. Fig. 6b and station location 10184 in Fig. 8). The observed sounding from Greifswald is shown here just for comparison; it has no influence on the icing diagnostic. The plotted curves of dewpoint temperature and temperature are quite similar. The model forecast humidity is greater than the observed values above $500 \mathrm{hPa}$ and the forecast temperature is too low by about $2 \mathrm{~K}$ between 600 and $400 \mathrm{hPa}$. Also there are slight deviations in the lower troposphere that are, however, not relevant for the icing diagnostic. Both soundings show the typical warm nose in the temperature curve between 960 and $820 \mathrm{hPa}(800 \mathrm{hPa}$ in the forecast). The observed warm nose is slightly more pronounced and the layer above ground is almost dry, in contrast to the forecast, which shows saturated air. Obviously the dry layer is not deep enough to evaporate the supercooled droplets as the surface station reports freezing rain. Summarizing we see that despite some departures in the observed and forecast soundings the icing algorithm is able to correctly describe the icing scenario. The freezing rain extends from the surface up to about $960 \mathrm{hPa}$, as indicated by the red bar on the right side in Fig. 7. For practical purposes ADWICE gives diagnostics for the surface as well as on flight levels. In Fig. 9a some areas of freezing rain are also seen to be present on flight level 25. There is also a zone of freezing rain over Finland that obviously does not reach the ground (cf. Fig. 6b). Note that surface stations in this area report mixed rain and snow (Fig. 4).

Despite the overforecast regions, we can state that ADWICE's first guess would have been able to issue a warning of freezing rain well in advance (about $8 \mathrm{~h}$ in this case taking into account operational processing and data distribution times of the LM model; see also section 


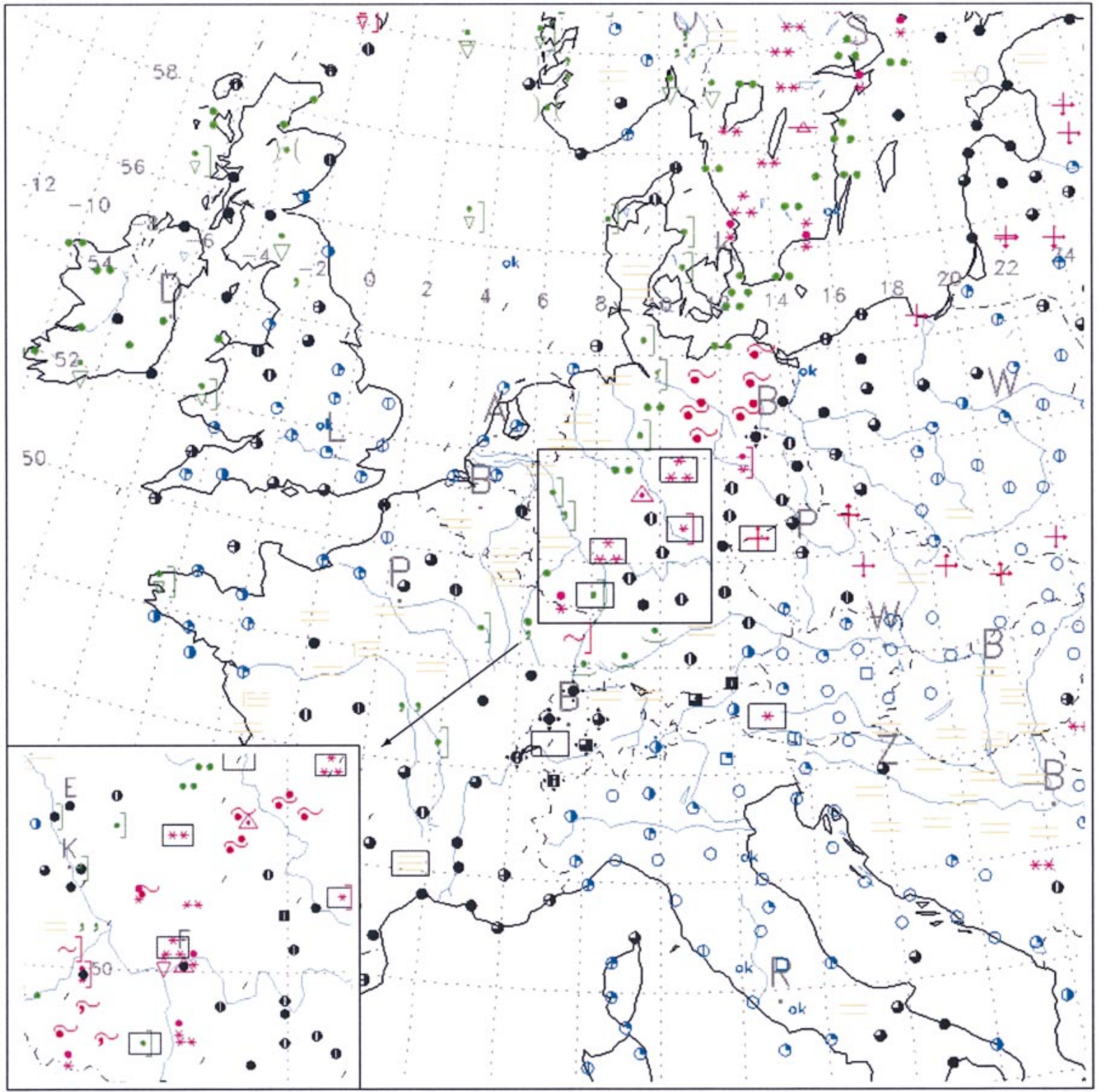

FIG. 4. Weather observations at 1200 UTC 23 Dec 1999 over Europe, extracted from MAP. Symbols are in standard WMO notation. Station circles are drawn in blue, if cloud cover is less than or equal to $4 / 8$ ("ok" means clearing and visibility okay for aviation), or in black, if cloud cover is more than 4/8. Capital letters mark the locations of capital cities: Paris, Dublin, London, Kopenhagen, Berlin, Warsaw, Prague, Vienna (W), Budapest, Bucarest, and Rome. Note that not all reporting stations are drawn to aid in visibility. Therefore, a subdomain encompassing the southwestern portion of the freezing-rain band over western Germany has been enlarged and copied to the lower-left corner of the figure. This subdomain includes all reports at this time.

6) before the actual occurrence and that also the position of the freezing-rain band was correctly forecast.

Let us now turn to the other icing regimes forecast and diagnosed for this time. At the surface the firstguess forecast exhibits yellow and blue areas indicating warm stratus and general icing, respectively (Fig. 6a). Surface observations reporting cloud cover of less or equal than 4/8 (cf. Fig. 4) reject most of the icing in these areas (Fig. 6b). Also over Lithunia where surface stations report total cloud cover (northeastern edge in
Fig. 4) general icing is rejected because the reported cloud height is well above the surface (more than 300 $\mathrm{m})$.

Inspection of the icing diagnostics on all flight levels from the surface up to $10000 \mathrm{ft}$ (for a complete list of all output levels, see section 6) shows that the freezing rain scenario extends up to flight level $2500 \mathrm{ft}$ (Fig. 9a) with some isolated patches appearing even on flight level $5000 \mathrm{ft}$ (not shown). Farther up, the icing types unstable (green) and general (blue) are predominant, as 


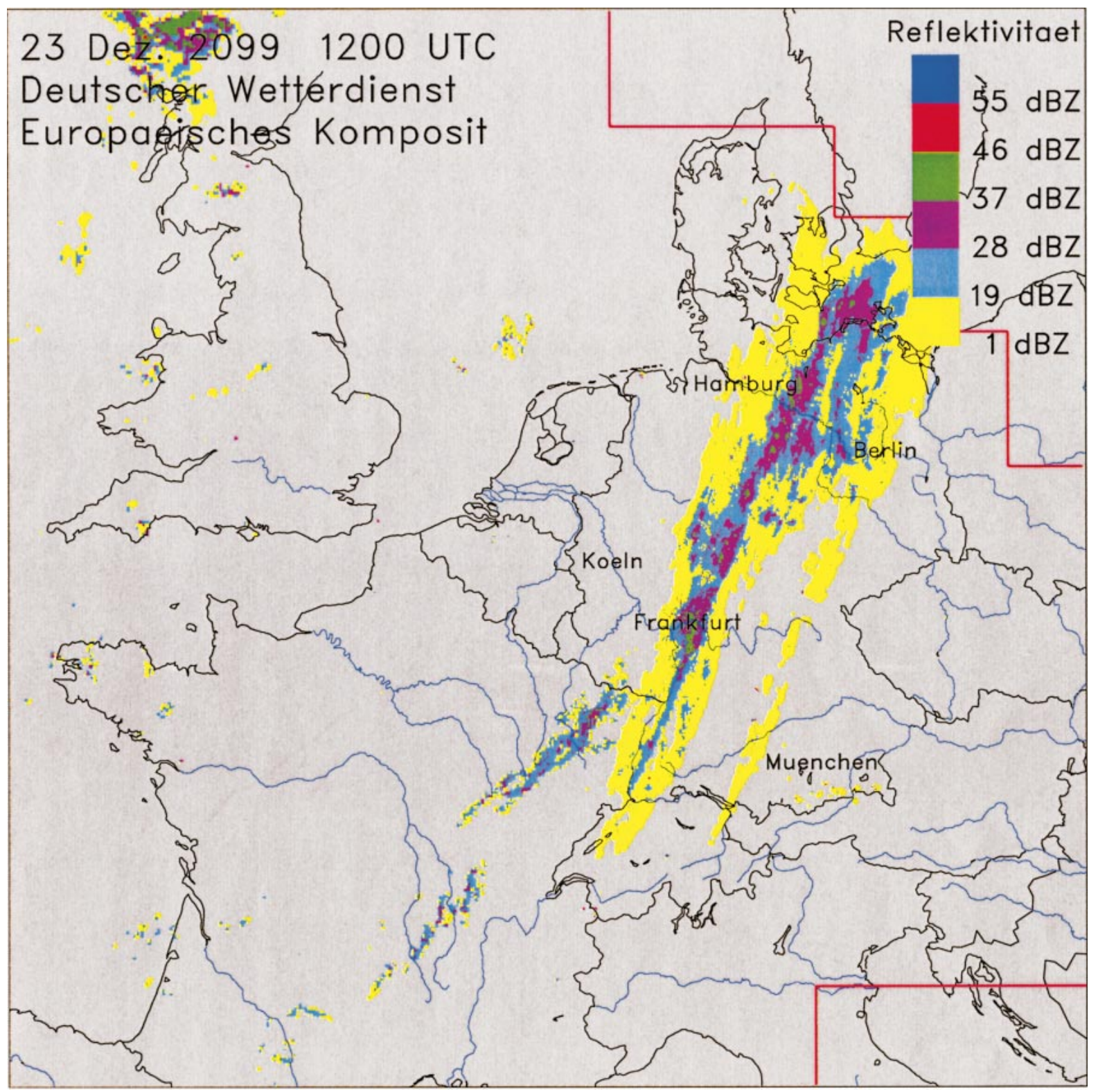

FIG. 5. European composite of radar reflectivity at 1200 UTC 23 Dec 1999 from DWD. Thin straight red lines in the right-hand corners at the top and bottom of the figure mark outer boundaries to the northeast and southeast of the composite, respectively.

seen, for example, on flight level $10000 \mathrm{ft}$ (Fig. 9b). The elongated band on level 100 extending southward from Sweden and farther westward over France is connected to the front and can be traced in the radar picture as well (Fig. 5) and in the observations of cloud cover (Fig. 4). In part of these areas the air is moist unstable, and supercooled droplets can exist within the clouds. In Fig. 7, green bars on the right-hand side of the sounding mark the levels where unstable type icing is diagnosed. Note the almost moist-adiabatic sounding between 650 and $550 \mathrm{hPa}$. Below $650 \mathrm{hPa}$ the sounding indicates stable stratification and therefore the color blue appears.
Unstable icing type is also diagnosed north of Ireland and general icing over southern Italy, which is also plausible given the respective surface and radiosonde observations (not shown).

Summarizing we can state that ADWICE is able to capture obvious icing features for this case and to distinguish different icing scenarios not only horizontally distributed but also in the vertical. However, we have to realize that without PIREPs a true verification of the different icing scenarios is not at hand. This is especially true as regards the icing severity given by the numbers in the plots. 

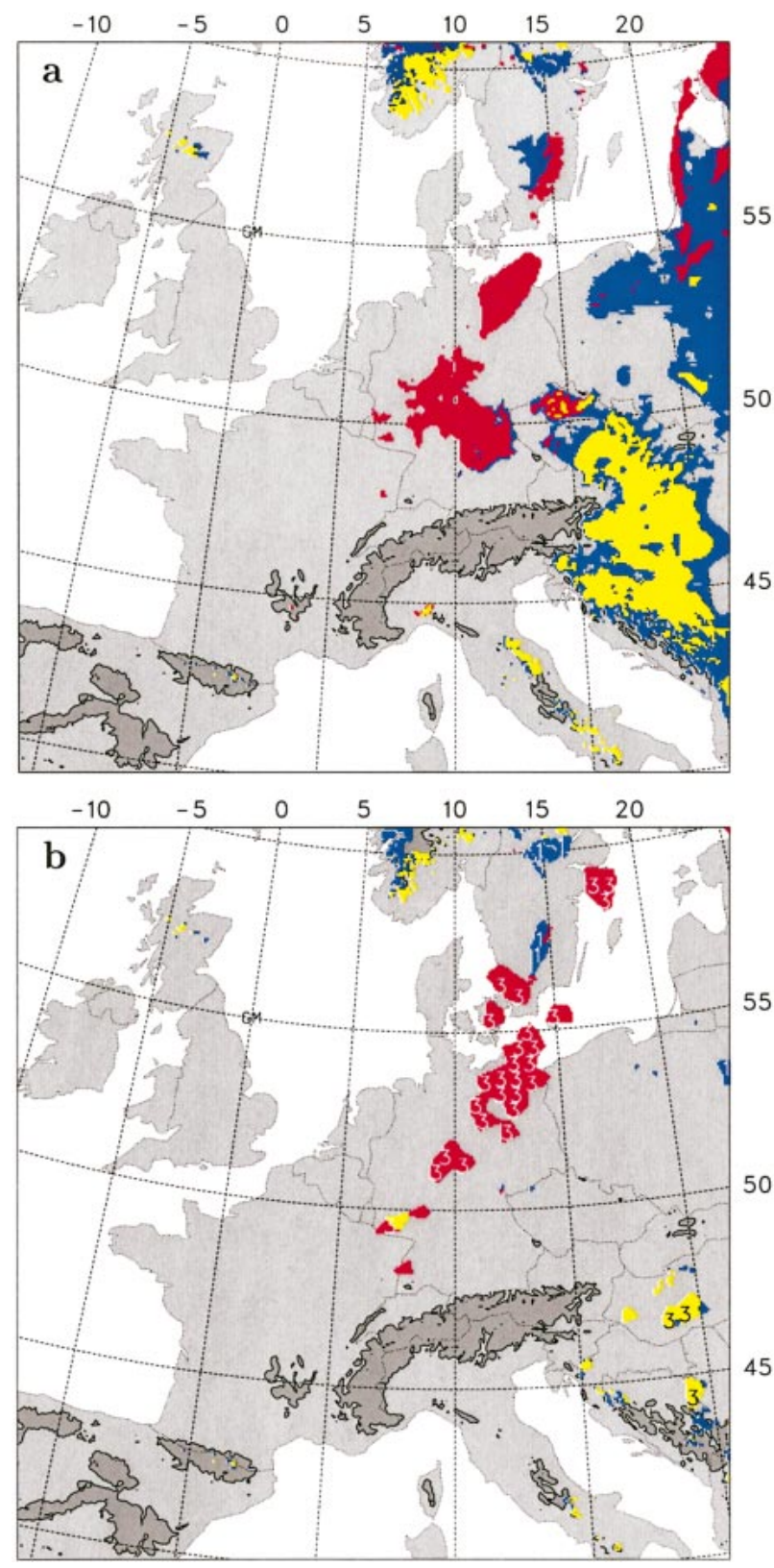

FIG. 6. Icing regions at the surface at 1200 UTC 23 Dec 1999 in the LM domain: freezing rain (red), warm stratus (yellow), unstable (green), and general (blue). (a) First guess as forecast by the LM after applying the NCAR/RAP algorithm, and (b) final icing with intensities 1 (light), 2 (moderate), or 3 (severe) after having applied the scenario correction and cloud correction.

\section{b. A case of severe icing on 13 November 2001}

On 13 November 2001 the large-scale flow over Europe is determined by a high pressure region (amplitude $1030 \mathrm{hPa}$ ) centered over Ireland and a low pressure system prevailing over central Italy (core pressure 995 $\mathrm{hPa}$ ). In the lower troposphere an almost saturated polar maritime air mass is directed toward the Alps from northerly directions. Figure 10 shows weather obser-

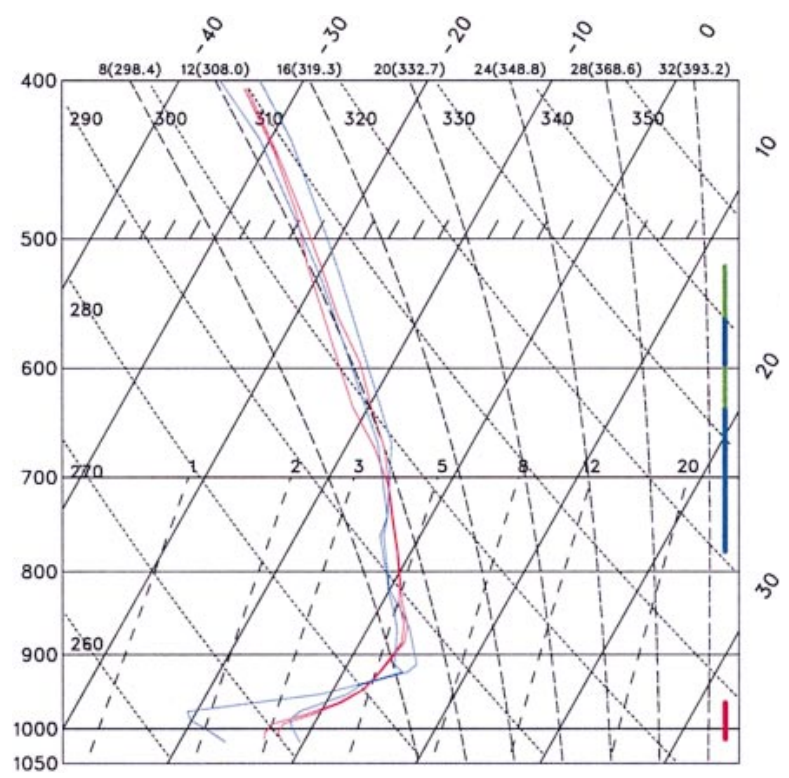

FIG. 7. Radiosonde observations of temperature and dewpoint temperature at Greifswald (blue lines) and the nearest LM sounding (red lines) at 1200 UTC 23 Dec 1999. Colored bars on the right-hand side mark diagnosed icing regimes in LM model layers (colors as in Fig. 6). For the location of this station refer to WMO identification 10184 in Fig. 8.

vations for 1200 UTC on 13 November 2001 for an area extending from northern Italy across the Alps to southern Germany. Also displayed are radar reflectivity (yellow and blue patches) and the locations of the radiosonde stations of Stuttgart (S), Munich (M), Vienna (W), Zagreb (Z), and the Italian stations Udine (just north of the Adriatic coast), Milano (in the Po valley), and Bo-

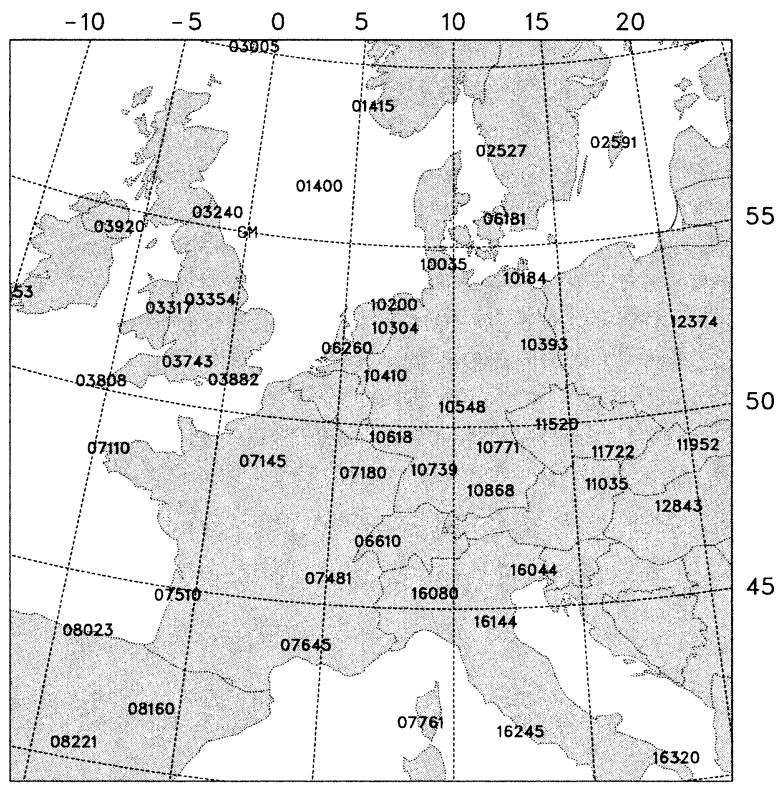

FIG. 8. Location of radiosonde stations within the LM domain (WMO codes). 

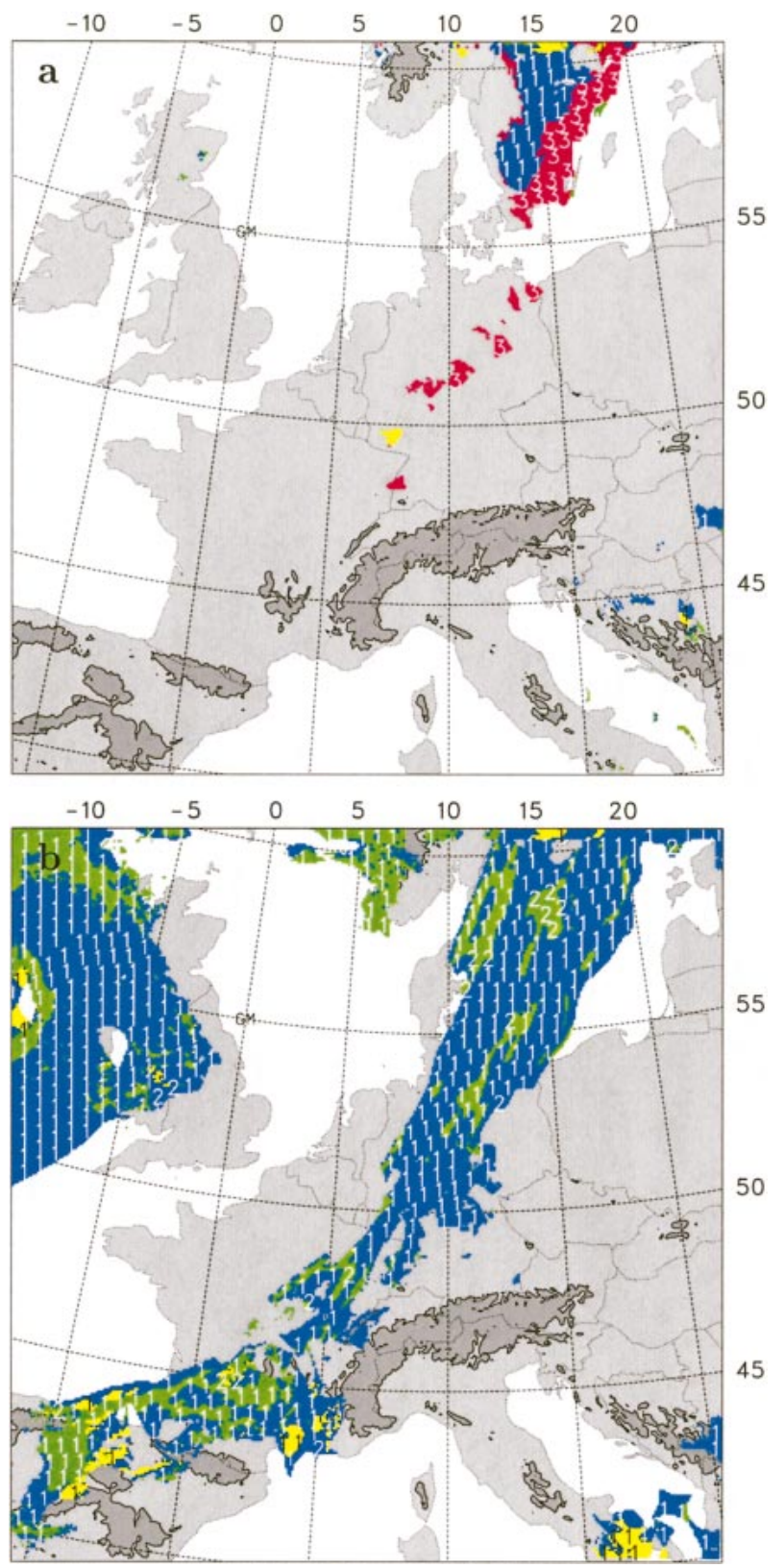

FIG. 9. Final icing diagnostic on flight level (a) $2500 \mathrm{ft}$ and (b) $10000 \mathrm{ft}$ for 1200 UTC 23 Dec 1999 in the LM domain: freezing rain (red), warm stratus (yellow), unstable (green), general (blue). Digits denote icing intensities 1 (light), 2 (moderate), or 3 (severe).

logna (southernmost station). Snow and light rain are reported from stations lying within the areas detected by radar in the intensities $1 \mathrm{dBZ}$ (yellow) and $19 \mathrm{dBZ}$ (blue). As there are moderate and severe icing PIREPs available (discussed below) close to the radiosonde observations of Stuttgart, Munich, and Bologna, we use these locations for discussion of the ADWICE performance.

The radiosonde observations from Munich (Fig. 11a; code number 10868 in Fig. 8) and Stuttgart (Fig. 11b; code number 10739 in Fig. 8) exhibit deep zones of almost saturated air within the temperature range from $0^{\circ}$ to $-24^{\circ} \mathrm{C}$ and $0^{\circ}$ to $-10^{\circ} \mathrm{C}$, respectively, in both the observations (blue lines for temperature and dewpoint temperature) and the 12-h forecast from the LM (red lines). In the Munich sounding the almost saturated air extends up to about $500 \mathrm{hPa}$. General icing (vertical blue lines on the right-hand side in the figures) has been diagnosed by the icing algorithms for the whole depth of the moist air mass. Whereas these soundings show stable stratified air north of the Alps within the flow from northerly directions, the sounding close to Bologna (Fig. 11c; San Pietro Capofiume, code number 16144 in Fig. 8), which represents the air mass north of the low pressure center, shows a complicated profile with varying stability and moist and dry zones. Note that the 12-h LM forecast (red lines) essentially captures this strongly varying profile. Accordingly the first-guess icing (colored bars on the right-hand side in Fig. 11c) shows different icing regimes depending on moisture and stability. In fact, three types of icing regimes appear: unstable (green), stratiform (yellow), and general (blue). The surface station at Stuttgart reports mixed drizzle and rain, and the one at Munich, mixed light rain and snow. At Stuttgart there is no radar reflectivity and at Munich, it is only at the lowest intensity: 1-19 dBZ (Fig. 10). Referring to Table 1 (fgss = ge) the scenario correction is such that it neither confirms nor rejects the first guess. Therefore, the first-guess icing is kept as the final icing diagnostic at these points. The Bologna station reports complete cloud cover. Therefore the first guess is not changed at this location either. Figure 12 shows the final icing diagnostic on flight level 10000 ft. Around the northern rim of the Alps general icing prevails (blue regions) as represented by the soundings from Munich and Stuttgart. Within other icing zones, unstable icing occurs (green) and there are some spots of warm stratus (yellow). Icing intensity is mostly diagnosed as light (1) except at some locations within the unstable regimes where moderate (2) is found.

Table 3 shows a list of PIREPs collected for this day together with the corresponding ADWICE diagnostics of icing intensity. Close to Munich there is one PIREP (D) of moderate icing at $110 \mathrm{hft}$ (hft $\equiv 100 \mathrm{ft}$; slightly above the 700-hPa level in Fig. 11a) and there is also one close to Stuttgart (E) with severe icing between 100 and $170 \mathrm{hft}$ (between approximately 700 and $500 \mathrm{hPa}$ in Fig. 11b). There is another PIREP (B) that is about $30 \mathrm{mi}$ south of the radiosonde station of Bologna, which reports moderate icing between flight levels 120 and $160 \mathrm{hft}$, that is, approximately between 650 and $550 \mathrm{hPa}$ in the radiosonde sounding of Bologna (Fig. 11c). It seems that the airplane has entered the top of a convective cell as the air mass between 800 and $650 \mathrm{hPa}$ in the sounding is moist unstable (blue temperature observation in Fig. 11c). The LM forecast temperature 


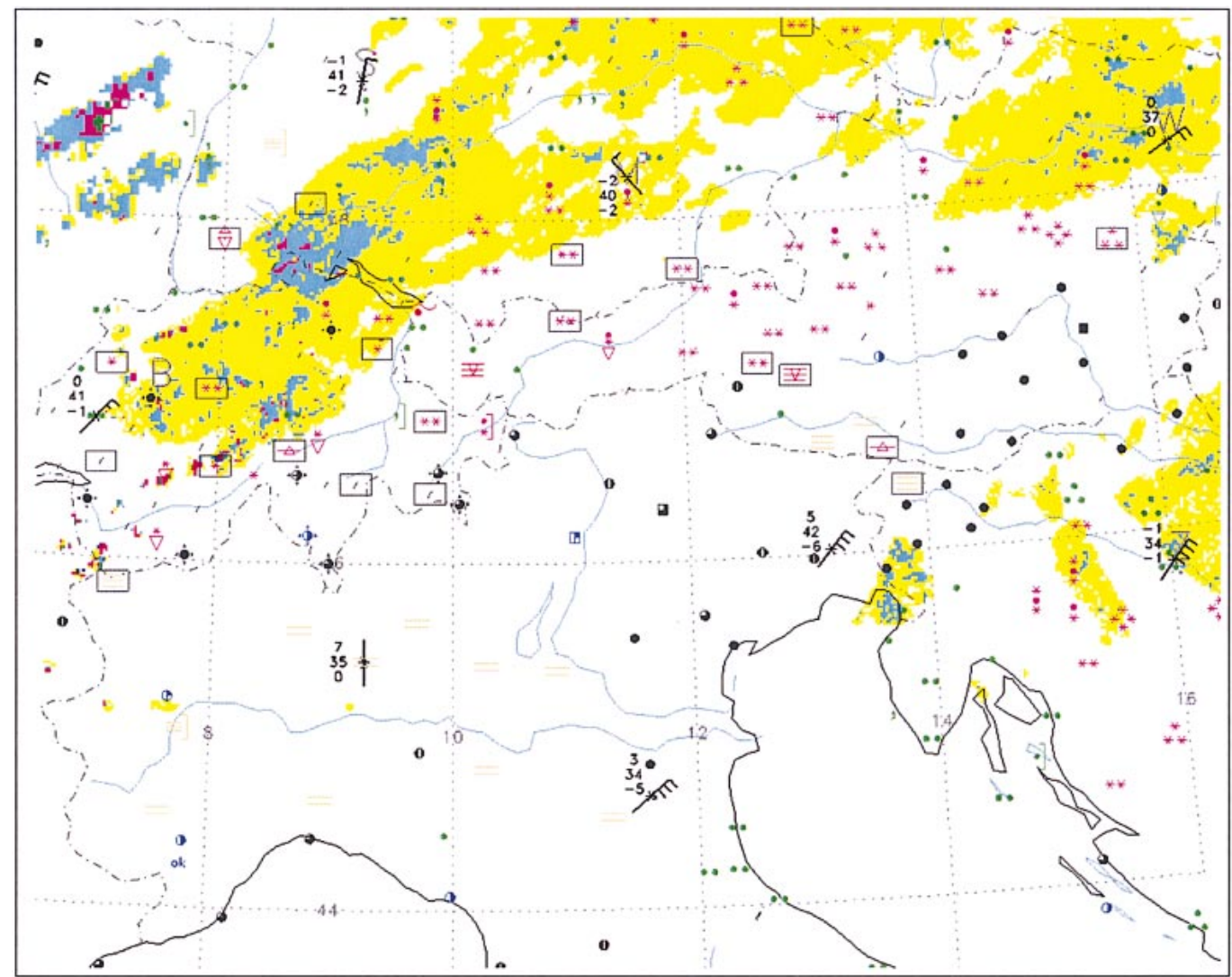

FIG. 10. Weather observations in standard notation at 1200 UTC 13 Nov 2001 overlaid with radar reflectivity (colors as in Fig. 5). Black wind barbs mark wind at $850 \mathrm{hPa}$ reported from radiosonde stations Stuttgart (S), Munich (M), Vienna (W), Zagreb (Z), Payerne [southwest of Bern (B)], and the Italian stations Udine (just north of the Adriatic coast), Milano (in the Po valley), and Bologna (southernmost station).

profile is moist unstable between about 730 and $660 \mathrm{hPa}$ and between 770 and $850 \mathrm{hPa}$. In between, a small stable layer prevents "unstable icing" from being diagnosed throughout this vertical extent; otherwise, the greencolored bars on the right-hand side in the sounding would be connected between 800 and $660 \mathrm{hPa}$. Recall, however, that the PIREP and the radiosonde location are $30 \mathrm{mi}$ (i.e., about seven grid distances) apart. Inspection of the LM sounding at the location of the PIREP shows unstable icing for a layer extending from 700 to almost $600 \mathrm{hPa}$, that is, between flight level 100 and 140, which is close to the reported icing range from flight level 120 to 160 . There are two more severe icing observations between 100 and $120 \mathrm{hft}(\mathrm{C})$ and between 90 and $120 \mathrm{hft}(\mathrm{F})$. In comparing these PIREPs to ADWICE fields we face two major problems. First, we have to recall that PIREPs are subjective estimations of icing severity and therefore they are not absolute measures of the icing intensity and, probably more important, given several reported flight heights or upper and lower bounds, we do not know whether the icing intensity accounts for the whole vertical extent between the levels reported or if it occurred only once or repeatedly be- tween those levels. Second, there is the reported problem with Eq. (1) of distributing available water vapor as condensed liquid water over several LM vertical layers. If we inspect the ADWICE output profiles at grid points corresponding to the PIREP locations, we find the values given in the two right-hand-most columns of Table 3. Here $\overline{\mathrm{LWC}}$ is the calculated liquid water content from Eq. (1) averaged over the LM model levels within the vertical extent of the icing zone as given by the respective PIREP report. For the average the arithmetic mean is taken. From Table 2 we see that all values are within the limits of the light category. Therefore, ADWICE underpredicts the icing severity when we average like this. However, if we add all the liquid water released within the vertical icing zone, the summed values achieve icing intensities in close agreement with the PIREPs. We even find severe icing in one case and a value of $1.10 \mathrm{~g} \mathrm{~m}^{-3}$ for another cases, which is close to the value of $1.33 \mathrm{~g} \mathrm{~m}^{-3}$ required for severe.

If we would take predicted cloud liquid water from the LM model for deducing icing severity, we would grossly fail. For almost all the points within the icing zones the cloud liquid water is close to zero. Only for 


\section{a}

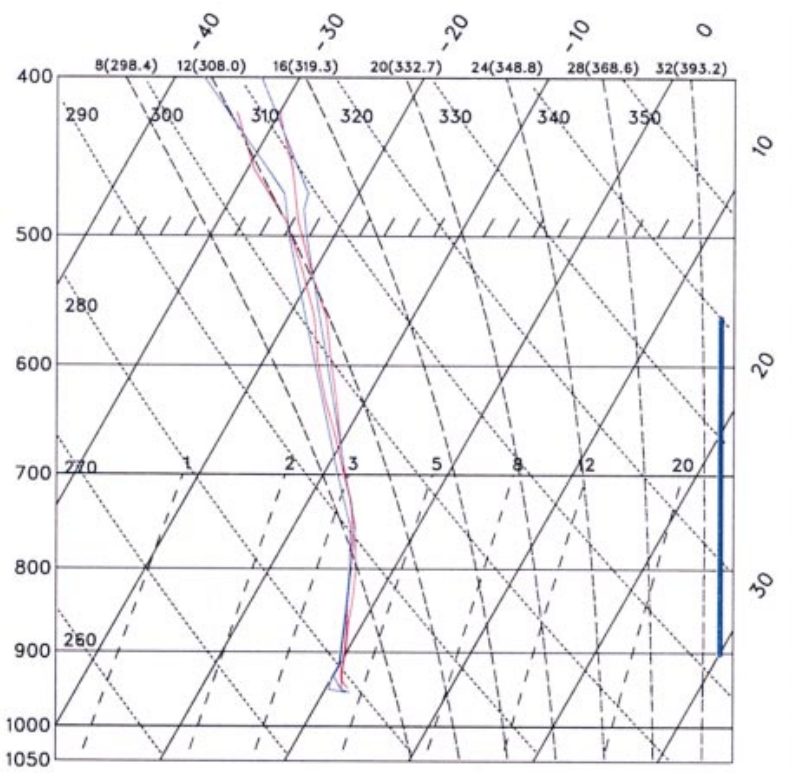

b

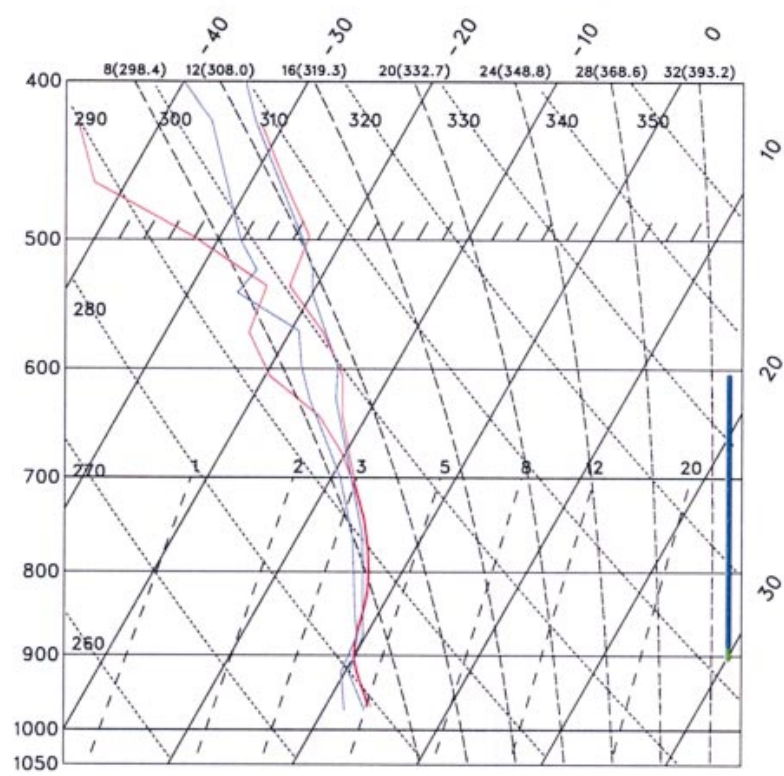

C

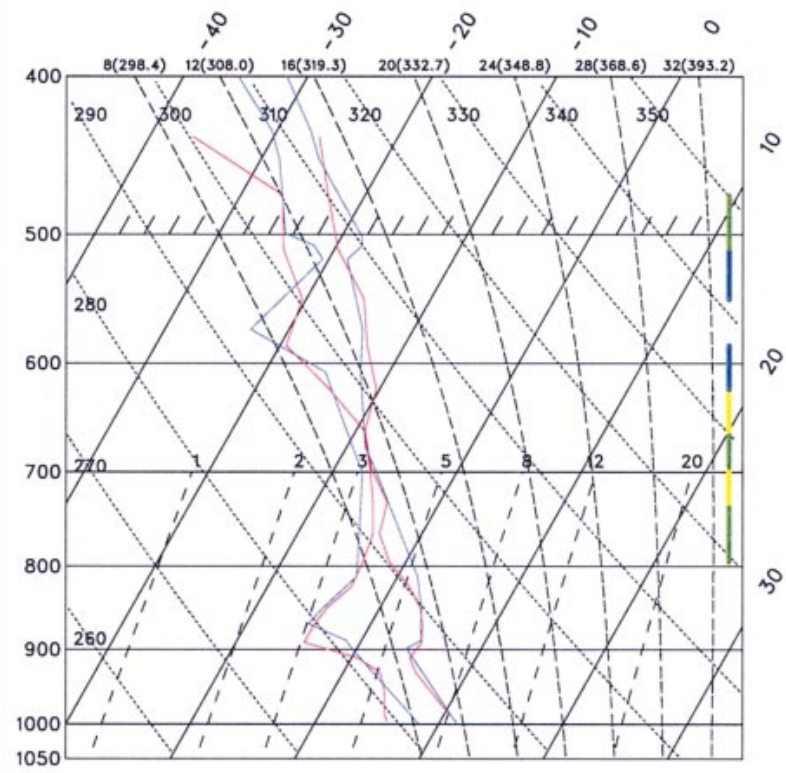

FIG. 11. Radiosonde observations of temperature and dewpoint temperature (blue lines in diagrams) at (a) Munich (WMO code 10868), (b) Stuttgart (10739), and (c) San Pietro Capofiume near Bologna (16144) together with nearest LM forecast soundings (red lines) after $12 \mathrm{~h}$ for 1200 UTC 13 Nov 2001. Colored bars on the right-hand side mark diagnose icing regimes in LM model layers (colors as in Fig. 6). For the location of these stations refer to Fig. 8 . the PIREP at $47^{\circ} 26^{\prime} \mathrm{N}$ and $11^{\circ} 56^{\prime} \mathrm{E}$ a value of $0.18 \mathrm{~g}$ $\mathrm{m}^{-3}$ is forecast for the respective level. This is an accord with the already mentioned problem of a proper representation of cloud physics processes in the LM model (and probably of other mesoscale models as well). Note that within the respective icing regions the forecast relative humidity is close to $100 \%$ (Figs. 11a, 11b) and that the far more realistic liquid water content calculated in ADWICE from Eq. (1) takes LM mixing ratios of water vapor as input. Summarizing for this case we can state that ADWICE diagnoses icing areas that are confirmed by six icing PIREPs at different locations north and south of the Alps. However, uncertainty remains with regard to comparing the icing intensity with PIREPs. 


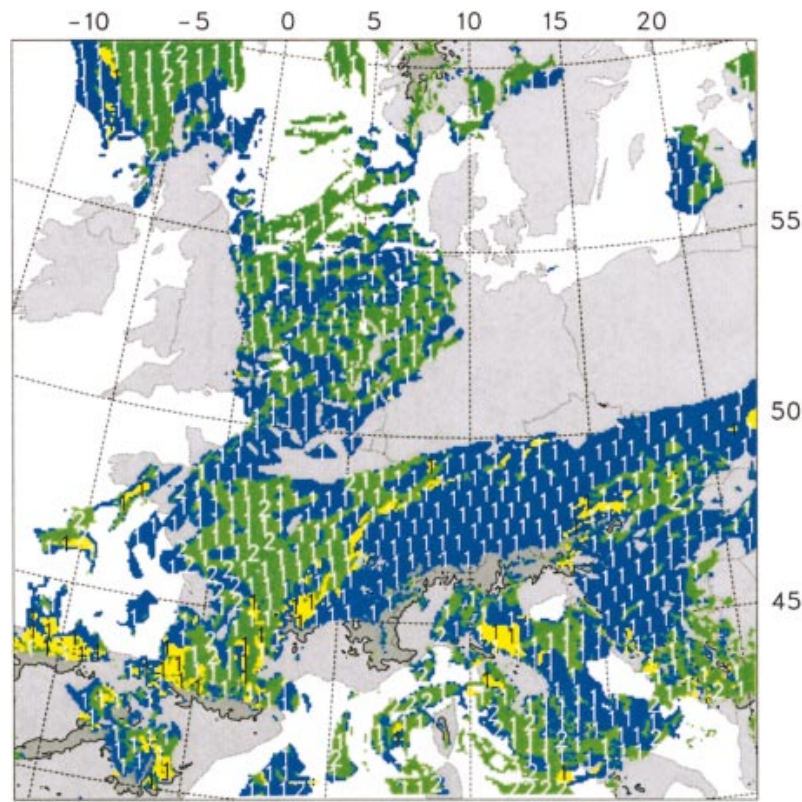

FIG. 12. Icing class and intensity on flight level $10000 \mathrm{ft}$ at 1200 UTC 13 Nov 2001. Coding as in Fig. 9.

\section{Use of satellite data}

It has been demonstrated by Thompson et al. (1997b) that satellite data can provide useful information for reducing diagnosed icing areas. At DLR, the Advanced Very High Resolution Radiometer (AVHRR) Processing Scheme over Clouds, Land and Ocean (APOLLO) cloud detection and analysis scheme has been developed (Kriebel et al. 1989). It discriminates among fully cloudy pixels, cloud-free pixels, and partially cloudy pixels with a pixel size of approximately $1 \mathrm{~km}^{2}$. Because it uses observations from the polar-orbiting National Oceanic and Atmospheric Administration (NOAA) satellites, which have only a few overpasses over Europe per day, the application of these data for ADWICE on a routine basis is not practical. However, as the intent is to apply the APOLLO system to Meteosat Second Generation (MSG) data as well, once it is available (launched on 28 August 2002). APOLLO can provide additional useful cloud information for ADWICE. For a test case on 19 April 2001 where an overpass of NOAA-14 at 1500 UTC was available, we used the information about cloudless pixels from APOLLO to cut out all icing grid points in a vertical column for respective LM grid points (not shown). In a similar way the APOLLO analysis can be used to cut out overforecast icing in regions where the forecast cloud-top temperature (CTT) is less than the observed CTT (forecast cloud top higher than observed). In addition to the data from the AVHRR instrument, MSG will also provide information about cloud phase, thus providing valuable information on icing conditions near cloud top where the maximum concentration of liquid water is sometimes found in embedded convection-type clouds (Hauf and Schröder 1998). The high reporting frequency (every $15 \mathrm{~min}$ ) together with the high spatial resolution (about $1 \mathrm{~km}$ ) of MSG data will enable the use of these data in a future version of ADWICE.

\section{Operational aspects}

During the months of January to May 2001 (phase 1) ADWICE was run operationally at DWD in Offenbach, Germany, and an updated version started to run in February 2002 (phase 2). During these testing phases ADWICE output has been evaluated by forecasters at the regional forecasting office at Frankfurt, which advises the aviation community and also receives PIREPs at real time. The experience gained from comparing ADWICE fields against actual weather situations and PIREP reports during phase 1 has substantially helped to identify obvious shortcomings in the ADWICE system. This has led to the new influence regions for SYNOP and METAR stations described in this study. Previously, a prescribed influence radius of $25 \mathrm{~km}$ had been set fixed to these stations, which led sometimes to quite "patchy" icing regions. In addition, the scenario correction was changed in various aspects. Figure 13 illustrates the timing of the processes during the course of the day. Two different procedures of ADWICE are distinguished: first guess (FG) and fusion (F). The FG procedure starts as soon as a forecast of the LM model (LM) is finished and gridded binary (GRIB) output is available in the DWD databank. This is about 0315 UTC

TABle 3. Pilot reports of icing on 13 Nov 2001 together with ADWICE diagnostics for icing intensity and liquid water content. Headers are time (UTC); lat $\left({ }^{\circ} \mathrm{N}\right)$; lon $\left({ }^{\circ} \mathrm{E}\right)$; min $\mathrm{H}$, reported minimum flight height (hft); max $\mathrm{H}$, reported maximum flight height (hft); mean, flight height (hft) if only one height reported or several varying only slightly in the vertical; PIREP, reported icing intensity from pilot; ADW $\overline{\mathrm{LWC}}$, icing intensity and liquid water content $\left(\mathrm{g} \mathrm{m}^{-3}\right)$ diagnosed from ADWICE for the reported flight level of the aircraft or mean value if PIREP includes lower- and upper-flight levels within which icing occurred; and ADW $\Sigma$ LWC, icing intensity and integrated liquid water content over all LM layers within reported vertical extent of icing.

\begin{tabular}{ccccccccccc}
\hline \hline Symbol & Time & Aircraft type & Lat $\left({ }^{\circ \prime}\right)$ & Lon $\left({ }^{\circ}\right)$ & Min H & Max H & Mean & PIREP & ADW $\overline{\text { LWC }}$ & ADW $\Sigma$ LWC \\
\hline A & 1120 & DO328 & 4726 & 1156 & & & 100 & LGT & LGT 0.11 & LGT 0.11 \\
B & 1140 & MD80 & 4402 & 1118 & 120 & 160 & & MOD & LGT 0.48 & MOD 0.96 \\
C & 1219 & ATR & 4855 & 0920 & 100 & 120 & & SEV & LGT 0.36 & MOD 1.10 \\
D & 1240 & & 4822 & 1148 & & & 110 & MOD & LGT 0.30 & LGT 0.30 \\
E & 1310 & B737 & 4841 & 0947 & 100 & 170 & & SEV & LGT 0.28 & SEV 1.41 \\
F & 1316 & DH8 & 4703 & 1600 & 90 & 120 & & SEV & LGT 0.41 & MOD 0.83 \\
\hline
\end{tabular}




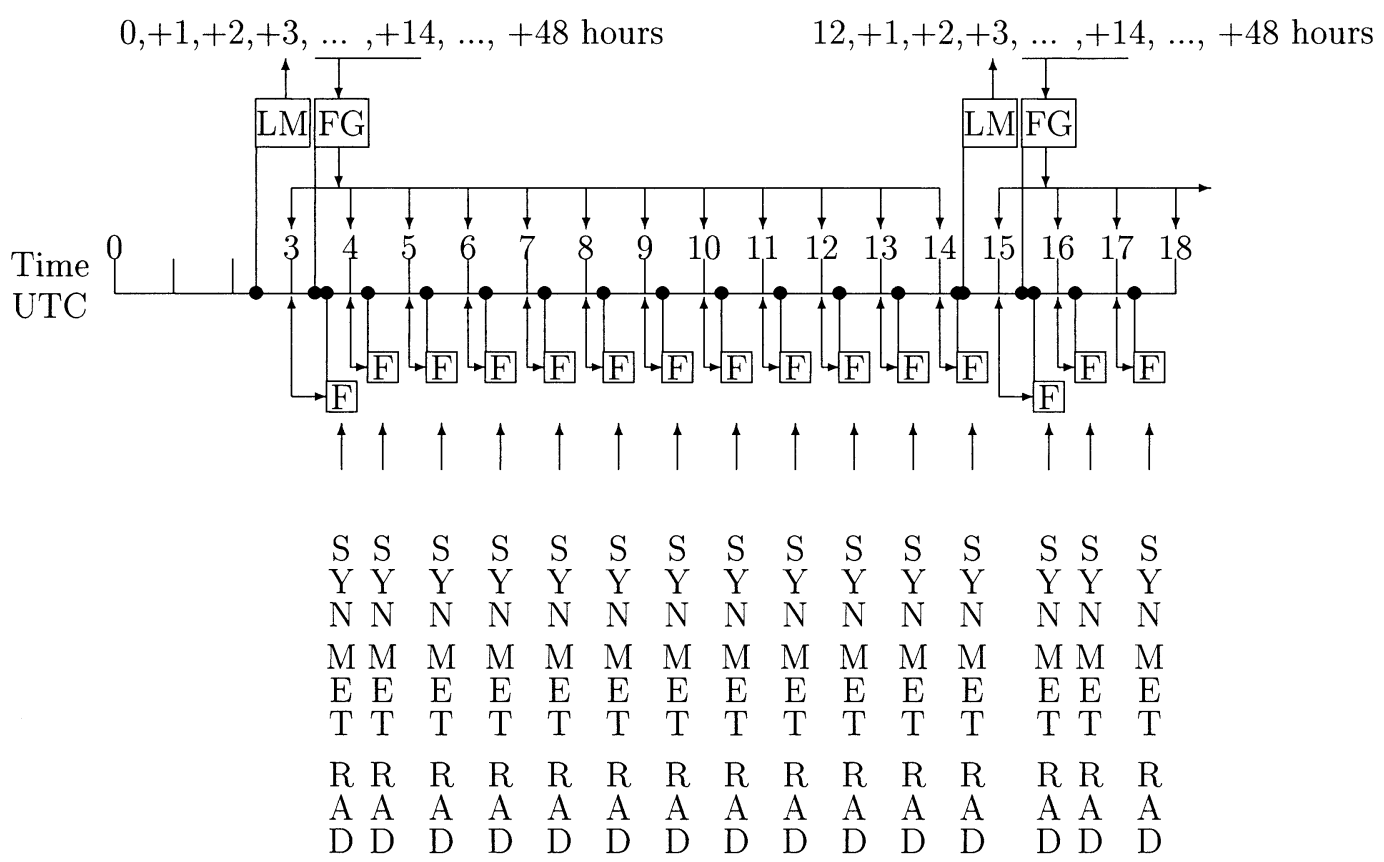

FIG. 13. ADWICE operational job timing: LM, Lokal Modell (DWD); FG, first-guess part of ADWICE, and F, fusion part of ADWICE. Bullets on the time axis mark the initialization time of the processes. Arrows indicate the data flow. SYN MET RAD stands for hourly observations of SYNOP, METAR, and radar data.

for the 0-h run of LM and 1515 UTC for the 12-h run. Then FG calculates the first guess icing grid derived from the NCAR/RAP algorithm (section $3 \mathrm{a}$ ) for $12 \mathrm{~h}$ in advance starting from 0300 and 1500 UTC, respectively. This process takes FG only a few minutes of CPU time on an SGI ORIGIN. The fusion procedure runs every hour as soon as SYNOP, METAR, and radar data are available for the full hour. METAR data are taken from reports during the previous $10 \mathrm{~min}$ only. Every fusion run outputs GRIB coded icing information on LM model levels as well as on the following pressure levels 1000, 950, 925, 900, 875, 850, 800, 750, 700, $650,600,550,500,400$, and $300 \mathrm{hPa}$ for representing flight levels $0,20,25,30,40,50,65,80,100,120,140$, $160,180,240$, and $300 \mathrm{hft}$. Graphics of the kind shown in this paper are produced online. As mentioned in section 5 satellite data are not yet taken into account operationally. As both job parts of ADWICE run within a few minutes of execution time, this setup make a timely icing forecast (the first guess) and icing diagnostic (after fusion run) possible without any unacceptable delays due to data processing and data dissemination.

\section{Summary and discussion}

Two icing events have been selected to help demonstrate the performance of the ADWICE system. Although the ADWICE icing fields appeared to be qualitatively correct with regard to the overall synoptic situation as given by surface, radiosonde, and PIREP ob- servations, the degree of over- or underforecasting can only be judged when many cases have been inspected carefully and verified against PIREPs. It is therefore intended to use the PIREPs collected during the testing campaigns of ADWICE both for further verification and tuning of the system. For example, we note that the selected thresholds used in the NCAR/RAP algorithm are somewhat uncertain, especially the humidity ranges, which might have to be adjusted to the numerical model in use. Whereas the LM has a grid distance of $7 \mathrm{~km}$, Thompson et al. (1997a) used the operational Eta (Mesinger et al. 1988) and MAPS Models (Benjamin et al. 1998), which had grid distances of 80 and $40 \mathrm{~km}$, respectively, at the time. Also different moist physics schemes in the models might affect the relative humidity choices in the algorithm. Furthermore, one might replace the simple yes-no flags used in the scenario correction by a more sophisticated fuzzy logic scheme. PIREPs available in real time could also be used as an additional data source. Also, as mentioned in section 5, observations of cloud coverage, cloud-top temperature, and cloud phase derived from satellite measurements will certainly bring new, valuable information into the ADWICE system.

From the architecture of the icing algorithms and the use of available observational data it is clear that the quality of the icing product depends very much on the first guess field and therefore on the quality of the LM forecast soundings. For example, a confirmed first guess of "warm stratus" by radar and surface observations signifies that icing conditions are quite likely to exist 
within the stratiform cloud and could therefore be given high probability. However, if this first guess is rejected by radar due to the observation of strong precipitation within the cloud, we are left with no definite information about the actual icing threat.

Another approach would be not to base the icing diagnosis on the first-guess icing fields, but first combine all available observations in order to identify cloudy and precipitating regions and afterward take the LM forecast fields into account. This approach is incorporated in the Integrated Icing Diagnostic Algorithm (IIDA; McDonough and Bernstein 1999). However, within IIDA, satellite data have a strong influence on the icing product and as long as we cannot use APOLLO operationally within ADWICE, an icing diagnosis based on surface and radar observations alone would be not complete. As mentioned in section 5, the intent is to change the APOLLO cloud detection and analysis tool for use with MSG data. Therefore, we will be able to use satellite data operationally for ADWICE after the availability of MSG data in 2003. The measuring channels of MSG may also allow for the deduction of the cloud phase, thus providing liquid water in cloud tops. In comparison to IIDA, ADWICE benefits from a higher station density for most parts in Europe as compared with the United States and also the LM model horizontal resolution is $7 \mathrm{~km}$ as compared with $40 \mathrm{~km}$ of the Rapid Update Cycle-2 (RUC-2) model. Note that in IIDA a distance of up to $125 \mathrm{~km}$ is taken for RUC-2 points as being influenced by surface observations when there are no nearby observations within a $40-\mathrm{km}$ distance.

Without question ADWICE will in the future automatically benefit from improvements made to the LM, especially with regard to data analysis, cloud physics, and grid resolution. More realistic cloud water forecasts from the LM could also eventually be used for diagnosing icing severity. At this stage however, diagnosing icing severity remains a problem not easily solved. The formula used in this setup of ADWICE has drawbacks but at least it provides better results than relying on cloud water forecasts that have been found to be very poor. An evaluation with all PIREPs collected during both testing phases might help to improve upon this issue. All in all, the data fusion concept followed in the design of ADWICE seems to be a step in the right direction, which can be extended with more sophisticated algorithms and new data sources.

Acknowledgments. In the first place we are grateful to Greg Thompson and his colleagues from the NCAR/ RAP team for making the icing algorithms available to us. We thank Martin Hagen from IPA/DLR for his support in decoding radar data, Hermann Mannstein and Sabine Rentsch from IPA/DLR for providing APOLLO cloud analyses, and Karl-Theodor Kriebel for fruitful discussions about the APOLLO system. We also thank Winfried Beer from IPA/DLR for his great and repeated help in all aspects of UNIX and SGI operation system maintenance, and data storage. Special thanks go to the DWD development team of MAP in Postdam, Sybille Haucke and Wolf Dietrich Polte, for their continuous support with regard to all concerns in installing and using the Meteorological Application and Presentation system and for their help in decoding GRIB data. Thanks are also due to the colleagues from the German Weather Service in Offenbach-Beate Löser, Wolfgang Löser, Robert Stanek, and Walter Ott-for providing meteorological data for the MAP workstation and their advice in using the databank at DWD. Numerous comments from two anonymous reviewers contributed greatly both in revising parts of the algorithms and improving the readability of the manuscript.

\section{APPENDIX}

\section{Derivation of Eq. (1)}

From the universal gas law we have for water vapor density $\rho_{v}$

$$
\rho_{v}=\frac{M_{v}}{R^{*}} \frac{p_{v}}{T}
$$

with molecular weight $M_{v}$ for water vapor, universal gas constant $R^{*}$, water vapor pressure $p_{v}$, and temperature $T$. Replacing $R^{*}$ with the individual gas constant $R$ and molecular weight $M_{a}$ for dry air gives

$$
\rho_{v}=\frac{1}{R T} \frac{M_{v}}{M_{a}} p_{v}=\frac{1}{R T} \frac{M_{v}}{M_{a}} \frac{p_{v}}{\left(p-p_{v}\right)}\left(p-p_{v}\right),
$$

with $p$ the pressure of moist air. Also we have for dry air

$$
\rho_{a}=\frac{M_{a}}{R^{*}} \frac{\left(p-p_{v}\right)}{T}
$$

and therefore we find for water vapor mixing ratio $m_{v}$

$$
m_{v}=\frac{\rho_{v}}{\rho_{a}}=\frac{M_{v}}{M_{a}} \frac{p_{v}}{\left(p-p_{v}\right)} .
$$

Inserting this into the equation for $\rho_{v}$ gives

$$
\rho_{v}=\frac{1}{R T} m_{v}\left(p-p_{v}\right) \approx \frac{1}{R T} m_{v} p .
$$

Assuming that the difference between water vapor mixing ratio at two vertical levels $i$ and $i+1$ ( $i$ increasing upward) within a cloud is totally condensed to water without freezing, precipitation, or diffusion, we get for the liquid water content

$$
\mathrm{LWC}=\rho_{v}(i)-\rho_{v}(i+1)=\frac{1}{R T}\left[m_{v}(i)-m_{v}(i+1)\right] p,
$$

with LWC in grams per cubic meter if $m_{v}$ is in grams per cubic meter. 


\section{REFERENCES}

Air Weather Service, 1980: Forecaster's guide on aircraft icing. Air Weather Service Tech. Rep. AWS/TR-80/001, Scott Air Force Base, IL, 55 pp.

Benjamin, S. G., J. M. Brown, K. J. Brundage, B. E. Schwartz, T. G. Smirnowa, and T. L. Smith, 1998: The operational RUC-2. Preprints, 16th Conf. on Weather Analysis and Forecasting, Phoenix, AZ, Amer. Meteor. Soc., 249-252.

Bernstein, B. C., 1996: A new technique for identifying locations where supercooled large droplets are likely to exist: The stovepipe algorithm. Preprints, 15th Conf. on Weather Analysis and Forecasting, Norfolk, VA, Amer. Meteor. Soc., 5-8.

- 2000: Regional and local influences on freezing drizzle, freezing rain, and ice pellets events. Wea. Forecasting, 15, 485-508

—, T. A. Omeron, F. McDonough, and M. K. Politovich, 1997: The Relationship between aircraft icing and synoptic-scale weather conditions. Wea. Forecasting, 12, 742-762.

- T. A. Omeron, M. K. Politovich, and F. McDonough, 1998: Surface weather features associated with freezing precipitation and severe in-flight icing. Atmos. Res., 46, 57-73.

Carrière, J.-M., S. Alquier, C. Le Bot, and E. Moulin, 1997: Statistical verification of forecast icing risk indices. Meteor. Appl., 4, 115130.

_ C. Lainard, C. Le Bot, and F. Robart, 2000: A climatological study of surface freezing precipitation in Europe. Meteor. Appl., 7, 229-238.

Cober, S. G., G. A. Isaac, and J. W. Strapp, 1995: Aircraft icing measurements in East Coast winter storms. J. Appl. Meteor., 34, $88-100$.

Doms, G., and U. Schättler, 1999: The nonhydrostatic Limited Area Model LM (Lokal Modell) of DWD. Part I: Scientific documentation. LM_F90 1.35, Deutscher Wetterdienst, Offenbach, Germany, $172 \mathrm{pp}$.

Federal Aviation Administration, 1974: Airworthiness standards, transport category airplanes. Part 25, Federal Aviation Regulations, Federal Aviation Administration, 158 pp. (Revised 1982.)

Forbes, G. S., Y. Hu, B. G. Brown, B. C. Bernstein, and M. K. Politovich, 1993: Examination of conditions in the proximity of pilot reports of icing during STORM-FEST. Preprints, Fifth Int. Conf. on Aviation Weather Systems, Vienna, VA, Amer. Meteor. Soc., 282-286.

Fuchs, W., and K.-P. Schickel, 1994: Computer assisted aircraft icing forecast: Progress and experiences. Atmos. Res., 34, 185-193.

Green, S., 1997: Icing problem is a serious threat for which the best solutions are years away. ICAO Journal, Jan./Feb. 97, 5-8, 25-26.

Guan, H., S. G. Cober, and G. A. Isaac, 2001: Verification of supercooled cloud water forecasts with in situ aircraft measurements. Wea. Forecasting, 16, 145-155.

Hauf, T., and R. Brown, 1998: Meteorological analysis of icing events. EURICE Tech. Rep. DL 14 TR 07, 28 pp.

— icing. Results from Research Flights in Germany, March 1997. Proc. Eighth Int. Workshop on Atmospheric Icing of Structures, Reykjavik, Iceland, RARIK-Iceland State Electricity, 67-77.

—, U. Finke, and F. Schröder, 1997: EURICE flight campaign. Flight program and instrumentation. DLR research aircraft CALM DO-228. EURICE Tech. Rep. DL 02 TR 001, 60 pp.

Hoffmann, H.-E., and J. Demmel, 1990: Analysis of three icing test flights reaching the aircraft-referred icing degree "severe." DLR-Forschungsbericht, Rep. 90-34, 114 pp.

Huffman, G. J., and G. A. Norman, 1988: The supercooled warm rain process and the specification of freezing precipitation. Mon. Wea. Rev., 116, 2172-2182.

Jeck, R. K., 1996: Representative values of icing-related variables aloft in freezing rain and freezing drizzle. Proc. FAA Int. Conf. on Aircraft In-flight Icing, Vol. II, Springfield, VA, DOT/FAA/ AR-96/81, 57-67.

Kane, T. L., B. G. Brown, and R. T. Bruintjes, 1998: Characteristics of pilot reports of icing. Preprints. 14th Conf. on Probability and Statistics in the Atmospheric Sciences, Phoenix, AZ, Amer. Meteor. Soc., 90-95.

Kriebel, K. T., R. W. Saunders, and G. Gesell, 1989: Optical properties of clouds derived from fully cloudy AVHRR pixels. Beitr. Atmos. Phys., 62, 165-171.

Kusch, W., and M. Pagoda, 1995: Meteorologisches Applikationsund Präsentationssytem für den dezentralen Wettervorhersagedienst (MAP). Promet, 24 (4), 85-95.

Leifeld, C., 1999: Das Nowcastingsystem ADWICE zur Diagnose und Vorhersage von vereisungsgefährdeten Lufträumen-Erste Validierung. M.S. thesis, Institute for Meteorology and Climatology, University Hannover, Hannover, Germany, 81 pp.

Lewis, W., 1947: A flight investigation of the meteorological conditions conducive to the formation of ice on airplanes. National Advisory Commitee for Aeronautics. Tech. Note 1393, 50 pp.

McCann, D. W., 1997: Five ways to produce supercooled drizzle drops. Preprints, Seventh Conf. on Aviation Range and Aerospace Meteorology, Long Beach, CA, Amer. Meteor. Soc., 9499.

McDonough, F., and B. Bernstein, 1999: Combining satellite, radar, and surface observations with model data to create a better aircraft icing diagnosis. Preprints, Eighth Conf. on Aviation, Range and Aerospace Meteorology, Dallas TX, Amer. Meteor. Soc., 467-471.

Mesinger, F., Z. I. Janjić, S. Ničković, D. Gavrilov, and D. G. Deavon, 1988: The step-mountain coordinate: Model description and performance for cases of Alpine lee cyclogenesis and for a case of an Appalachian redevelopment. Mon. Wea. Rev., 116, 1493-1518.

Mingione, G., 1998: EURICE: work-package 1 final report. CIRATR-97156, Centro Italiano Ricerche Aerospaziali, 38 pp.

Nicholls, S., 1987: A model of drizzle growth in warm, turbulent, stratiform clouds with a mixed layer model. Quart. J. Roy. Meteor. Soc., 123, 165-186.

Politovich, M. K., 1989: Aircraft icing caused by large supercooled droplets. J. Appl. Meteor., 28, 856-868.

- and B. C. Bernstein, 1995: Production and depletion of supercooled liquid water in a Colorado winter storm. J. Appl. Meteor., 34, 2631-2648.

Rauber, R. M., and A. Tokay, 1991: An explanation for the existence of supercooled water at the top of clouds. J. Atmos. Sci., 48, $1005-1023$.

—_ L. S. Olthoff, M. K. Ramamurthy, and K. E. Kunkel, 2000: The relative importance of warm rain and melting processes in freezing precipitation events. J. Appl. Meteor., 39, 1185-1195.

Reisner, J., R. M. Rasmussen, and R. T. Bruintjes, 1998: Explicit forecsting?? of supercooled liquid water in winter storms using the MM5 mesoscale model. Quart. J. Roy. Meteor. Soc., 124, 1071-1107.

Sand, W. R., W. A. Cooper, M. K. Politovich, and D. L. Veal, 1984: Icing conditions encountered by a research aircraft. J. Climate Appl. Meteor., 23, 1427-1440.

Schultz, P., and M. K. Politovich, 1992: Toward the improvement of aircraft-icing forecasts for the continental United States. Wea. Forecasting, 7, 491-500.

Strapp, J. W., R. A. Stuart, and G. A. Isaac, 1996: A canadian climatology of freezing precipitation and a detailed study using data from St. John's, Newfoundland. Proc. FAA In. Conf. on Aircraft In-flight Icing, Vol. II, Springfield, VA, DOT/FAA/AR96/81, 45-55.

Stuart, R. A., and G. A. Isaac, 1999: Freezing precipitation in Canada. Atmos.-Ocean, 37, 87-102.

Thompson, G., R. T. Bruintjes, B. G. Brown, and F. Hage, 1997a: Intercomparison of in-flight icing algorithms. Part I: WISP94 real-time icing prediction and evaluation program. Wea. Forecasting, 12, 878-889.

, T. F. Lee, and R. Bullock, 1997b: Using satellite data to reduce the spatial extent of diagnosed icing. Wea. Forecasting, 12, 185190. 